NISSUNA UMANA INVESTIGAZIONE SI PUO DIMANDARE VERA SCIENZIA S'ESSA NON PASSA PER LE MATEMATICHE DIMOSTRAZIONI LEONARDO DA VINCI

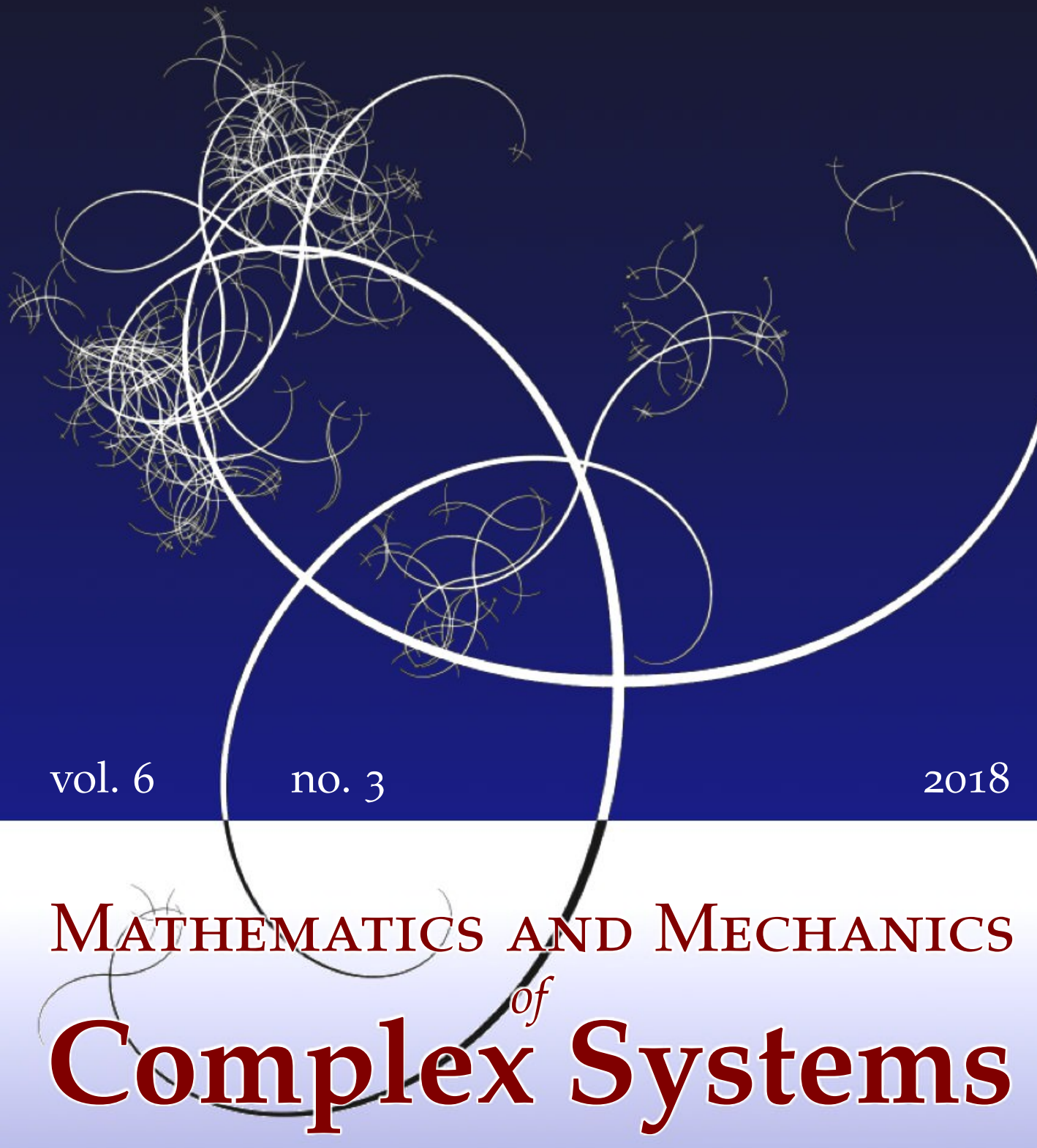

Houssam Abdoul-AnZIZ ANd PierRe SEPPECHER

STRAIN GRADIENT AND GENERALIZED CONTINUA OBTAINED BY HOMOGENIZING FRAME LATTICES 


\title{
STRAIN GRADIENT AND GENERALIZED CONTINUA OBTAINED BY HOMOGENIZING FRAME LATTICES
}

\author{
Houssam Abdoul-Anziz And Pierre SePpecher
}

\begin{abstract}
We determine the effective behavior of periodic structures made of welded elastic bars. Taking into account the fact that flexural and torsional stiffnesses are much smaller than the extensional one, we bypass classical homogenization formulas and obtain totally different types of effective energies. We work in the framework of linear elasticity. We give different examples of 2D or 3D microstructures which lead to generalized 1D, 2D, or 3D continua like the Timoshenko beam, Mindlin-Reissner plate, strain gradient, or Cosserat or micromorphic continua.
\end{abstract}

1. Introduction

2. Initial problem, description of the geometry, and notation 216

3. Homogenization result 222

4. Explicit computation of the homogenized stiffness matrices 225

5. Examples 230

6. Conclusion 244

Acknowledgments $\quad 245$

$\begin{array}{ll}\text { References } & 245\end{array}$

\section{Introduction}

Composite materials have proved to be so useful in structural design that homogenization techniques have received considerable attention over the past few decades both in mechanics and mathematics. More recently researchers realized that homogenization of composites made of very highly contrasted materials could lead to exotic effective behaviors. On the other hand the new manufacturing processes, which allow for extremely fine designs, gave birth to the new research field of metamaterials (or architected materials).

From the mathematical point of view, asymptotic homogenization of periodic media is now well founded. It consists of taking into account the fact that the size of the periodic cell is much smaller than the characteristic size of the considered

\section{Communicated by Pierre Suquet.}

MSC2010: 35B27, 74Q05, 78M40.

Keywords: strain gradient, generalized continua, homogenization, lattices, $\Gamma$-convergence. 
sample and of passing to the limit when the ratio $\varepsilon$ between these two lengths tends to zero. This problem has been widely studied in static or dynamic cases, for conduction or elasticity problems, when the cell is made of a material with varying properties [Bensoussan et al. 1978; Sánchez-Palencia 1980; Allaire 1992; Oleŭnik et al. 1992]. Whenever the elastic energy of the material is a convex functional of the displacement field - and that is the case here where only linear elasticity is considered - a formula (see for instance [Allaire 1992, (3.6)] or [Milton 2002, p. 10]) gives the effective (i.e., limit) behavior of the medium in terms of a local minimization problem set in a rescaled cell. In this approach one lets $\varepsilon$ tend to zero alone, while all other parameters of the system remain fixed. However, in many cases some other small parameters are present and the relative convergence speeds are crucial for the effective behavior of the material. The case when the cell is made of materials with very different properties is called "high contrast homogenization" [Cherednichenko et al. 2006]. Closely related is the case (which could be called the "infinitely high contrast" case) when holes are present (see for instance [Tartar 2009, Chapter 16]). But a small parameter can also derive from strong anisotropy or from geometric considerations. It is known that the effective behavior can then strongly differ [Pideri and Seppecher 1997; Bouchitté and Bellieud 2002; Bellieud and Gruais 2005; Bellieud et al. 2016; Bellieud 2017] from the initial behavior of the materials of which the structure is made. The first results in this direction were dealing with conduction problems, and a nonlocal limit energy was found [Tartar 1989; Khruslov 1991]. We are more interested by limit energies involving higher derivatives than the initial ones. Indeed materials with such energies are seldom found in nature [Barbagallo et al. 2017a; 2017b] and are expected to have a very special behavior [dell'Isola et al. 2015a; Eringen 2001; Mindlin 1965]. Their most distinctive feature is that they do not enter the framework of Cauchy stress theory (the internal mechanical interactions are not described by a Cauchy stress tensor) [dell'Isola et al. 2015b; 2016b; 2017]. However, such models are frequently used for regularizing the singularities which may arise in fracture, plasticity, interfaces, etc. (see for instance [Triantafyllidis and Aifantis 1986; Polizzotto and Borino 1998; Aifantis 1992; 1999; Mazière and Forest 2015; Sciarra et al. 2007; Yang and Misra 2012]).

Here we deal with static linear elasticity. In this framework a general closure result [Camar-Eddine and Seppecher 2003] states that all regular enough objective quadratic energies can be obtained through homogenization of highly contrasted media. In particular energies depending on the second gradient of the displacement (or equivalently on the strain gradient) or nonlocal energies [Bellieud and Gruais 2005] like energies associated to generalized continua [Forest 1999] can be obtained. But the result stated in [Camar-Eddine and Seppecher 2003] does not provide any reasonably applicable procedure for designing a microstructure with these exotic effective properties. 
Note that we are interested here in the actual effective (i.e., limit) energy and not in corrections at order $\varepsilon$ of a classical effective energy. The controversy about the sign of such corrective terms [Allaire et al. 2016; Le 2015] shows that they are difficult to interpret and to apply. Moreover, the fact that these corrective terms are present in conduction problems as well as in elasticity problems while it has been proved that no second gradient effect can appear in the limit energy for conductivity [Camar-Eddine and Seppecher 2002] shows that they are a very different notion.

A few structures have been described with a second gradient effective energy. Many of them [Pideri and Seppecher 1997; Bouchitté and Bellieud 2002; Bellieud et al. 2016; Bellieud 2017; Briane and Camar-Eddine 2007] lead to a couplestress model, that is, to an energy depending only on the gradient of the skewsymmetric part of the gradient of the displacement [Toupin 1962; 1964; Mindlin 1963; Mindlin and Tiersten 1962; Bouyge et al. 2002]. Some discrete structures [Alibert et al. 2003; Alibert and Della Corte 2015; Boutin 1996; Seppecher et al. 2011; Boutin et al. 2017] lead to a more general second gradient energy.

In a recent paper [Abdoul-Anziz and Seppecher 2018] we have provided the first rigorous homogenization result in continuous elasticity which led to a general second gradient energy. We have considered periodic structures made by a single very stiff linear elastic material and void. The geometry of the structure consists of connected slender bars. They are so slender that the ratio of the section of these bars with respect to the size of the cell is comparable to $\varepsilon$. We have been able to prove that the $2 \mathrm{D}$ elasticity problem was, as expected, asymptotically similar to a frame lattice whose bars have a much smaller flexural stiffness than extensional stiffness. Then we have established a general formula for computing the effective energy of the medium. This result differs from the ones given in [Martinsson and Babuška 2007b; 2007a; Gonella and Ruzzene 2008] where very similar discrete systems are studied. The point is that, in these works, the orders of magnitude of the different types of interactions are assumed, as is generally done (see [Martinsson and Babuška 2007b, Remark 7.5], [Meunier et al. 2012, (2.7)], or [Braides and Gelli 2002]) not to interfere with the homogenization asymptotic process. In [Pastukhova 2005] or [Zhikov and Pastukhova 2003] the authors have assumed, like we do, that the ratio of the section of the bars with respect to the size of the cell is of order $\varepsilon$, but they considered like in [Zhikov 2002] a too soft material for obtaining generalized continuum limits.

In this paper, we start with a discrete lattice and extend the homogenization result of [Abdoul-Anziz and Seppecher 2018] to dimension 3. We also study concomitant homogenization and reduction of dimension in order to describe beam or plate models. We precisely describe the algebraic computation needed for making explicit the effective behavior of the considered lattices. Then we explore the wide variety of models which can be obtained. We feel that these examples provide academic 
microscopic mechanisms enlightening the behavior of generalized materials. Our homogenization formula is a tool, which was up to now missing, for explaining how the strain or microdeformation can propagate in a strain gradient material or in a micromorphic material, respectively.

The paper is organized as follows. In Section 2 we fix the notation and the way of describing lattices. In Section 3 we recall the homogenization result and show that it can be recovered by using a formal expansion procedure. In Section 4 we present the algebraic computation needed for making explicit the effective energy in a sufficiently detailed way for enabling the reader to follow (and eventually check) the Octave/MATLAB package that we provide in [Abdoul-Anziz and Seppecher 2017]. Section 5 is devoted to the description of many examples, leading successively to beams, membranes, plates, and 3D materials. We recover classical models like Euler or Timoshenko beams, the Cosserat model for membranes, or the KirchoffLove or Mindlin-Reissner plate, but we also get strain gradient models with the possibility of mixing different effects.

\section{Initial problem, description of the geometry, and notation}

2.1. The frame lattice. In the physical space $\mathbb{R}^{3}$, we consider a periodic discrete lattice (see an example in Figure 1) defined by

- a bounded open domain $\underline{\Omega} \subset \mathbb{R}^{3}$,

- a small dimensionless parameter $\varepsilon$ which we will let tend to zero (this parameter compares the size of the periodic cell $\varepsilon Y$ of the lattice with the size of the macroscopic domain $\underline{\Omega}$ ),

- a prototype cell containing a finite number $K$ of nodes, the positions of which are denoted $y_{s} \in \mathbb{R}^{3}, s \in\{1, \ldots, K\},{ }^{1}$ and

- a family of $N$ independent periodicity vectors $t_{\alpha}, \alpha \in\{1, \ldots, N\}$, with $1 \leq$ $N \leq 3$.

The case $N=3$ corresponds to standard 3D homogenization while the cases $N=2$ and $N=1$ correspond to 3D-2D and 3D-1D concomitant homogenization and reduction of dimension, respectively. They lead to plate or beam models. ${ }^{2}$

We assume with no loss of generality that the vector space $\mathbb{R}^{N}$ spanned by the vectors $t_{\alpha}$ coincides with the space spanned by the $N$ first vectors of the canonical basis $\left(e_{1}, e_{2}, e_{3}\right)$ of the physical space. The intersection of $\underline{\Omega}$ with $\mathbb{R}^{N}$ is denoted $\Omega$, and we assume (a simple choice of the unit length) that its $N$-dimensional volume satisfies $|\Omega|=1$.

\footnotetext{
${ }^{1}$ Note that lower-dimension cases $y_{s} \in \mathbb{R}$ or $y_{s} \in \mathbb{R}^{2}$ can be treated by simply embedding $\mathbb{R}$ or $\mathbb{R}^{2}$ in $\mathbb{R}^{3}$.

${ }^{2}$ Note that 2D-2D and 2D-1D are also treated by using the previous remark.
} 


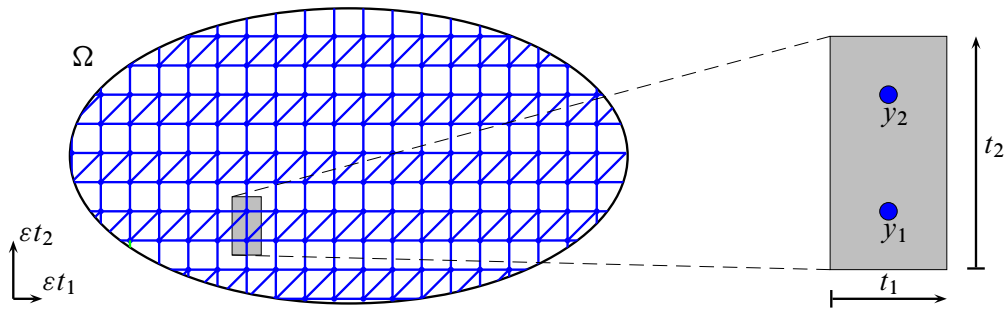

Figure 1. A prototype cell in a typical planar geometry when $K=2$ and $N=2$.

For $I=\left(i_{1}, \ldots, i_{N}\right) \in \mathbb{Z}^{N}$, consider the points $y_{I, S}^{\varepsilon}:=\varepsilon\left(y_{S}+i_{1} t_{1}+\cdots+i_{N} t_{N}\right)$. We use $y_{I}^{\varepsilon}:=(1 / K) \sum_{s=1}^{K} y_{I, S}^{\varepsilon}$ as a reference point in the cell $I$. The nodes of the considered lattice are those nodes which lie sufficiently inside the domain $\underline{\Omega}$ : more precisely the nodes $y_{I, s}^{\varepsilon}$ with $s \in\{1, \ldots, K\}$ and $I \in \mathcal{I}^{\varepsilon}$ where

$$
\mathcal{I}^{\varepsilon}:=\left\{I: y_{I}^{\varepsilon} \in \underline{\Omega}, d\left(y_{I}^{\varepsilon}, \partial \underline{\Omega}\right)>\sqrt{\varepsilon}\right\} .
$$

The cardinal of this set, denoted $N^{\varepsilon}$, is of order $\varepsilon^{-N}$. In the sequel, for any field $\Phi_{I, s}$ defined at the nodes of the structure, we will denote by $\sum_{I} \Phi_{I, s}$ the mean values

$$
\sum_{I} \Phi_{I, s}:=\frac{1}{N^{\varepsilon}} \sum_{I \in \mathcal{I}^{\varepsilon}} \Phi_{I, s} \sim \varepsilon^{N} \sum_{I \in \mathcal{I}^{\varepsilon}} \Phi_{I, s} .
$$

For any fixed cell $I$, the number of closest neighboring cells is $3^{N}-1$. Counting the cell $I$ itself, these cells are the cells $I \pm p$ with $p \in \mathcal{P}$ (the cardinal of $\mathcal{P}$ is $\left.n=\left(3^{N}+1\right) / 2\right)$. When $N=1,2$, or 3 we can choose

$\mathcal{P}:=\{0,1\}$,

$\mathcal{P}:=\{(0,0),(1,0),(0,1),(1,1),(1,-1)\}$,

$\mathcal{P}:=\{(0,0,0),(1,0,0),(0,1,0),(1,1,0),(1,-1,0),(0,0,1),(0,1,1),(0,1,-1)$,

$$
(1,0,1),(1,0,-1),(1,1,1),(1,1,-1),(1,-1,1),(1,-1,-1)\},
$$

respectively. In all cases, respecting the order given above, we identify $\mathcal{P}$ with $\{1, \ldots, n\}$. For any $p=\left(p_{1}, \ldots, p_{N}\right) \in \mathcal{P}$, we denote by $\boldsymbol{p}:=p_{1} t_{1}+\cdots+p_{N} t_{N}$ the corresponding vector so that $y_{I+p, s}^{\varepsilon}=y_{I, s}^{\varepsilon}+\varepsilon \boldsymbol{p}$.

For any pair of distinct nodes $\left(y_{I, s}^{\varepsilon}, y_{I+p, s^{\prime}}^{\varepsilon}\right)$, we denote

$$
\ell_{p, s, s^{\prime}}:=\varepsilon^{-1}\left\|y_{I+p, s^{\prime}}^{\varepsilon}-y_{I, s}^{\varepsilon}\right\|, \quad \tau_{p, s, s^{\prime}}:=\left(y_{I+p, s^{\prime}}^{\varepsilon}-y_{I, s}^{\varepsilon}\right) /\left\|y_{I+p, s^{\prime}}^{\varepsilon}-y_{I, s}^{\varepsilon}\right\| .
$$

2.2. Mechanical interactions. To make precise the mechanical structure we are considering, we have to make precise the mechanical interactions between the nodes. The structures we want to model are periodic grids or frames made of 
welded elastic bars. Essentially, the nodes behave like small rigid bodies and the interactions between these bodies can be divided in two parts. The extensional stiffness of one bar controls the relative displacements of its extremity nodes in the direction of the bar while the flexural and torsional stiffnesses control the relative rotations of its extremity nodes and the difference between these rotations and the global rotation of the bar.

Without loss of generality we assume that a cell is interacting only with its closest neighbors: indeed we can always choose a prototype cell large enough for this assumption to become true. Taking into account the symmetry, it is enough to fix the interactions between the nodes of cell $I$ and between the nodes of cell $I$ and half of its closest neighbors $I+p$ with $p \in \mathcal{P}$ (see Figure 2).

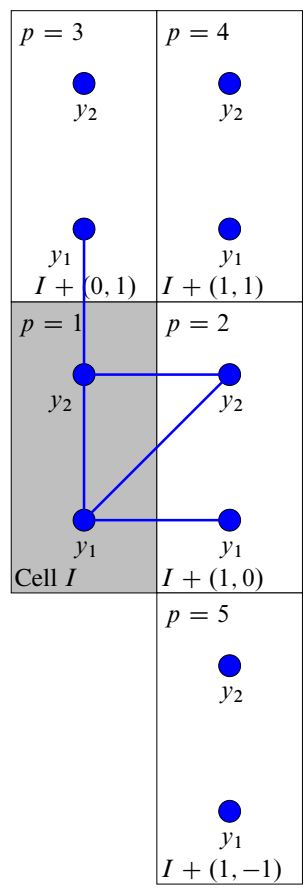

Figure 2. Fixing the interactions between a cell and its neighbors: here $K=2$ and $N=2$ and nonvanishing interactions are represented by blue lines, namely an internal $(p=1)$ interaction between nodes $y_{1}$ and $y_{2}$ $\left(a_{1,1,2} \neq 0\right)$, three interactions with the cell on the right $(p=2)$ between node $y_{1}$ and nodes $y_{1}$ and $y_{2}$ and between nodes $y_{2}\left(a_{2,1,1} \neq 0, a_{2,1,2} \neq 0\right.$, and $\left.a_{2,2,2} \neq 0\right)$, and a last interaction with the cell above $\left(a_{3,2,1} \neq 0\right)$. In this example there is no interaction with neighbor cells $p=4$ nor $p=5$, but such interactions could have been considered. Interactions with the cells below or on the left do exist. Owing to periodicity they do not need to be fixed. 
- Extensional interactions. These interactions between the nodes of the lattice are determined by $n K^{2}$ nonnegative coefficients $a_{p, s, s^{\prime}}$. We introduce the set of multi-indices corresponding to all pairs of nodes in interaction:

$$
\mathcal{A}:=\left\{\left(p, s, s^{\prime}\right): p \in \mathcal{P}, 1 \leq s \leq K, 1 \leq s^{\prime} \leq K, a_{p, s, s^{\prime}} \neq 0\right\} .
$$

For any displacement field $U$ of the lattice, that is, a vector field $U_{I, s}$ defined on $\mathcal{I}^{\varepsilon} \times\{1, \ldots, K\}$, we call the "extension" between nodes $(I, s)$ and $\left(I+p, s^{\prime}\right)$ the quantity

$$
\left(\rho_{U}\right)_{I, p, s, s^{\prime}}:=\frac{U_{I+p, s^{\prime}}-U_{I, s}}{\varepsilon} \cdot \tau_{p, s, s^{\prime}} .
$$

The extensional energy of the lattice has the form

$$
\begin{aligned}
E^{\varepsilon}(U) & :=\varepsilon^{-2} \sum_{I} \sum_{\left(p, s, s^{\prime}\right)} \frac{a_{p, s, s^{\prime}}}{2}\left(\rho_{U}\right)_{I, p, s, s^{\prime}}^{2} \\
& =\varepsilon^{-2} \sum_{I} \sum_{\left(p, s, s^{\prime}\right) \in \mathcal{A}} \frac{a_{p, s, s^{\prime}}}{2}\left(\rho_{U}\right)_{I, p, s, s^{\prime}}^{2} .
\end{aligned}
$$

- Flexural/torsional interactions. We attach to each node $(I, s)$ of the structure a rigid motion: in addition to the displacement $U_{I, s}$, each node is endowed with a rotation vector ${ }^{3} \theta_{I, s}$. Let us introduce the vector

$$
\left(\alpha_{U}\right)_{I, p, s, s^{\prime}}:=\tau_{p, s, s^{\prime}} \times \frac{U_{I+p, s^{\prime}}-U_{I, s}}{\varepsilon \ell_{p, s, s^{\prime}}} .
$$

As mechanical interactions need to be objective (i.e., invariant when adding both a constant value $\Phi$ to the field $\theta_{I, s}$ and the field $\Phi \times y_{I, s}^{\varepsilon}$ to the displacement field $\left.U_{I, s}\right)$, flexural/torsional interaction between nodes $(I, s)$ and $\left(I+p, s^{\prime}\right)$ has to be a positive quadratic form of two vectors $\theta_{I, s}-\left(\alpha_{U}\right)_{I, p, s, s^{\prime}}$ and $\theta_{I+p, s^{\prime}}-\left(\alpha_{U}\right)_{I, p, s, s^{\prime}}$. It can be represented by a nonnegative $6 \times 6$ matrix. ${ }^{4}$

Thus, flexural/torsional interactions are determined by $3 n K^{2}$ nonnegative matrices $B_{p, s, s^{\prime}}, C_{p, s, s^{\prime}}, D_{p, s, s^{\prime}}$ whose elements are $3 \times 3$ matrices, so that the flexural energy reads

$$
\begin{aligned}
& F^{\varepsilon}(U, \theta):= \\
& \qquad \sum_{I} \sum_{\left(p, s, s^{\prime}\right)}\left[\left(\theta_{I, s}\right.\right. \\
& \left.\quad+\left(\alpha_{U}\right)_{I, p, s, s^{\prime}}\right) \cdot \frac{B_{p, s, s^{\prime}}}{2} \cdot\left(\theta_{I, s}-\left(\alpha_{U}\right)_{I, p, s, s^{\prime}}\right) \\
& +\left(\theta_{I, s}-\left(\alpha_{U}\right)_{I, p, s, s^{\prime}}\right) \cdot C_{p, s, s^{\prime}} \cdot\left(\theta_{I+p, s^{\prime}}-\left(\alpha_{U}\right)_{I, p, s, s^{\prime}}\right) \\
& \left.+\left(\theta_{I+p, s^{\prime}}-\left(\alpha_{U}\right)_{I, p, s, s^{\prime}}\right) \cdot \frac{D_{p, s, s^{\prime}}}{2} \cdot\left(\theta_{I+p, s^{\prime}}-\left(\alpha_{U}\right)_{I, p, s, s^{\prime}}\right)\right] .
\end{aligned}
$$

\footnotetext{
${ }^{3}$ Remember that we are in the framework of linear elasticity and that rotations are represented by skew-symmetric matrices which can be identified by vectors.

${ }^{4}$ Note that objectivity implies also that the rank of this matrix cannot exceed 5.
} 
We assume that flexural/torsional interaction is present only in conjunction with extensional interaction:

$$
a_{p, s, s^{\prime}}>0 \Longleftrightarrow\left(\begin{array}{ll}
B_{p, s, s^{\prime}} & C_{p, s, s^{\prime}} \\
C_{p, s, s^{\prime}}^{t} & D_{p, s, s^{\prime}}
\end{array}\right)>0
$$

Our choice of the order of magnitude of these interactions needs some comment. We first emphasize that speaking of the order of magnitude of the stiffness of a structure makes sense only if we compare it to some force. In other words, making an assumption about the elastic rigidity is equivalent to making an assumption about the order of magnitude of the applied external forces.

Our aim is to consider structures for which classical homogenization would lead to a degenerated material. As usual a rescaling process is needed if one wants to capture a finite limit energy. Different assumptions can be made which correspond to different experiments. This is not surprising: the reader accustomed for instance to the $3 \mathrm{D}-2 \mathrm{D}$ or $3 \mathrm{D}-1 \mathrm{D}$ reduction of models for plates or beams knows that changing the assumptions about the order of magnitude of the elasticity stiffness of the material drastically changes the limit model. If the structure cannot resist some applied forces (like a membrane cannot resist transverse forces), it may resist them after a suitable scaling of the material properties (like the membrane model is replaced by the Kirchhoff-Love plate model). Simultaneously some mobility may disappear (like the Kirchhoff-Love plate becomes inextensible). Our choice of the order of magnitude of the extensional interactions means that the applied external forces are not sufficient to significantly extend the bonds between nodes. On the other hand, we have assumed that the flexural rigidities were much smaller than the extensional ones. This is unavoidable when considering structures in which mechanical interactions are due to slender parts. The chosen order of magnitude $\left(\varepsilon^{0}\right)$ is critical. Other cases can be deduced from our results by letting in a further step $\left(b_{p}, c_{p}, d_{p}\right)$ tend to zero or to infinity. The assumption that the ratio between bending and extension stiffnesses is comparable to the homogenization small parameter $\varepsilon$ is essential: we emphasize that one cannot capture all interesting asymptotic effects by homogenizing the structure in a first step and letting the ratio bending stiffness/extension stiffness tend to zero in a second step; see [Martinsson and Babuška 2007b].

Example. Assume the lattice consists of slender cylinders joining the interacting nodes. Assume that all the cylinders have a circular basis of radius $r^{\varepsilon}=\beta \varepsilon^{2}$ and are made of a homogeneous isotropic elastic material with Young modulus $\mathcal{Y}$ and Poisson coefficient $v$. Extension, bending, and torsion rigidities of an elastic cylindrical bar of radius $r^{\varepsilon}$ are classical results of mechanics [Germain 1973]. Integrating along the bar, one can deduce the values of the interactions due to the elasticity of a bar of length $\ell^{\varepsilon}$. We get 


$$
\begin{aligned}
B_{p, s, s^{\prime}}=D_{p, s, s^{\prime}} & =a_{p, s, s^{\prime}}\left(f \mathrm{Id}+(t-f) \tau_{p, s, s^{\prime}} \otimes \tau_{p, s, s^{\prime}}\right), \\
2 C_{p, s, s^{\prime}} & =a_{p, s, s^{\prime}}\left(f \mathrm{Id}-(2 t+f) \tau_{p, s, s^{\prime}} \otimes \tau_{p, s, s^{\prime}}\right)
\end{aligned}
$$

with

$$
a_{p, s, s^{\prime}}=\frac{\mathcal{Y} \pi \beta^{2} \varepsilon^{7} N^{\varepsilon}}{\ell_{p, s, s^{\prime}}}, \quad f=\beta^{2}, \quad t=\frac{\beta^{2}}{4(1+v)} .
$$

This case satisfies our assumptions as soon as one assumes that the Young modulus of the material is of order $\varepsilon^{N-7}$. Note that $f$ and $t$ are of order 1 and thus $a_{p, s, s^{\prime}}, B_{p, s, s^{\prime}}$, and $C_{p, s, s^{\prime}}$ have the same order. Indeed the difference of order between extensional and flexural/torsional stiffnesses, due to the slenderness $\beta \varepsilon$ of the bars, has already been taken into account by the factor $\varepsilon^{-2}$ introduced in the definition of $E^{\varepsilon}$.

Example. The case of a 2D lattice can also be treated in our framework. It is enough to fix $y_{s, 3}=0$ for all $s \in\{1, \ldots, K\}$ and $t_{\alpha, 3}=0$ for all $\alpha \leq N \leq 2$ and to focus only on planar displacements $U_{I, s, 3}=0$ and $\theta_{I, s, 1}=\theta_{I, s, 2}=0$ at all nodes. Let us assume that the nodes are linked by slender rectangles of thickness $\beta \varepsilon^{2}$. Textbooks in mechanics give the extension and bending rigidities of a slender rectangle. We still can use the matrices $B_{p, s, s^{\prime}}, C_{p, s, s^{\prime}}$, and $D_{p, s, s^{\prime}}$ defined in the previous example but modifying $a_{p, s, s^{\prime}}$ and $f$ in

$$
a_{p, s, s^{\prime}}=\frac{\mathcal{Y} \beta \varepsilon^{3} N^{\varepsilon}}{\ell_{p, s, s^{\prime}}}, \quad f=\frac{4 \beta^{2}}{3} .
$$

Note that $t$ plays no role in this example. This case satisfies again our assumptions as soon as one assumes that the Young modulus of the material is of order $\varepsilon^{N-5}$.

- Boundary conditions. We do not intend to study the way the different boundary conditions which could be imposed to our lattices pass to the limit. The richness [dell'Isola and Seppecher 1997; Seppecher et al. 2011] of the boundary conditions associated to generalized continua is such that trying to describe them in a general way is a real challenge. On the other hand, we cannot adopt the frequently used Dirichlet boundary conditions: indeed the lattices we consider generally present in the limit some inextensibility constraint and Dirichlet boundary conditions could lead to a trivial set of admissible deformations. So we consider here only free boundary conditions. So, in order to ensure uniqueness of the equilibrium solution, we impose a zero mean rigid motion:

$$
\sum_{I} \frac{1}{K} \sum_{s=1}^{K} U_{I, s}=0, \quad \sum_{I} \frac{1}{K} \sum_{s=1}^{K} \theta_{I, s}=0 .
$$

- Connectedness. We are not interested in structures made of different unconnected 
parts: we assume that mechanical interactions make a connected network. This has to be checked before using our results. This checking is generally obvious but is actually difficult to automate [Babuška and Sauter 2004].

\section{Homogenization result}

In a recent paper [Abdoul-Anziz and Seppecher 2018] we have rigorously derived the model (7)-(8) from a 2D linear elastic problem by analyzing the behavior of the slender substructures: we have then identified the effective energy through a $\Gamma$ convergence theorem (for a simple definition of this notion the reader can refer to [Braides 2002] or [Dal Maso 1993]) using tools of double scale convergence (see [Nguetseng 1989] or [Allaire 1992]). This has been done in the 2D-2D case. The extension of the proof to other dimensions does not need new arguments. We will provide here neither the proof which can be found in [Abdoul-Anziz and Seppecher 2018] nor the technical but straightforward extension to other dimensions. The goal of this paper is to explore the diversity of possible limit models. However, for the readers who do not desire to enter into the mathematical developments of [Abdoul-Anziz and Seppecher 2018], we show below that formal expansions of the kinematic variables actually give the right effective energy.

Assume that there exist smooth enough functions $\left(u, v_{s}, w_{s}, \theta_{s}\right)$ (for any $s \in$ $\{1, \ldots, K\})$ such that

$$
\begin{aligned}
U_{I, s}^{\varepsilon} & :=u\left(y_{I}^{\varepsilon}\right)+\varepsilon v_{s}\left(y_{I}^{\varepsilon}\right)+\varepsilon^{2} w_{s}\left(y_{I}^{\varepsilon}\right)+o\left(\varepsilon^{2}\right), \\
\theta_{I, S}^{\varepsilon} & :=\theta_{s}\left(y_{I}^{\varepsilon}\right)+o(1) .
\end{aligned}
$$

Then

$$
\begin{array}{r}
U_{I+p, s^{\prime}}^{\varepsilon}-U_{I, s}^{\varepsilon}=\varepsilon \nabla u\left(y_{I}^{\varepsilon}\right) \cdot \boldsymbol{p}+\frac{\varepsilon^{2}}{2} \nabla \nabla u\left(y_{I}^{\varepsilon}\right) \cdot \boldsymbol{p} \cdot \boldsymbol{p}+\varepsilon \\
\left(v_{s^{\prime}}\left(y_{I}^{\varepsilon}\right)+\varepsilon \nabla v_{s^{\prime}}\left(y_{I}^{\varepsilon}\right) \cdot \boldsymbol{p}-v_{s}\left(y_{I}^{\varepsilon}\right)\right) \\
+\varepsilon^{2}\left(w_{s^{\prime}}\left(y_{I}^{\varepsilon}\right)-w_{s}\left(y_{I}^{\varepsilon}\right)\right)+o\left(\varepsilon^{2}\right)
\end{array}
$$

and thus

$$
\begin{aligned}
\varepsilon^{2} E^{\varepsilon}\left(U^{\varepsilon}\right) & =\sum_{I} \sum_{\left(p, s, s^{\prime}\right) \in \mathcal{A}} \frac{a_{p, s, s^{\prime}}}{2}\left(\frac{U_{I+p, s^{\prime}}^{\varepsilon}-U_{I, s}^{\varepsilon}}{\varepsilon} \cdot \tau_{p, s, s^{\prime}}\right)^{2} \\
& =\sum_{I} \sum_{\left(p, s, s^{\prime}\right) \in \mathcal{A}} \frac{a_{p, s, s^{\prime}}}{2}\left(\left(\nabla u\left(y_{I}^{\varepsilon}\right) \cdot \boldsymbol{p}+v_{s^{\prime}}\left(y_{I}^{\varepsilon}\right)-v_{s}\left(y_{I}^{\varepsilon}\right)\right) \cdot \tau_{p, s, s^{\prime}}\right)^{2}+o(1) \\
& =\int_{\Omega} \sum_{\left(p, s, s^{\prime}\right) \in \mathcal{A}} \frac{a_{p, s, s^{\prime}}}{2}\left(\left(\nabla u(x) \cdot \boldsymbol{p}+v_{s^{\prime}}(x)-v_{s}(x)\right) \cdot \tau_{p, s, s^{\prime}}\right)^{2} d x+o(1) \\
& =\bar{E}\left(v, \eta_{u}\right)+o(1)
\end{aligned}
$$


where we define for any functions $v_{s}$ and $\eta_{p, s}$

$$
\bar{E}(v, \eta):=\int_{\Omega} \sum_{p, s, s^{\prime}} \frac{a_{p, s, s^{\prime}}}{2}\left(\left(\eta_{p, s^{\prime}}(x)+v_{s^{\prime}}(x)-v_{s}(x)\right) \cdot \tau_{p, s, s^{\prime}}\right)^{2}
$$

and

$$
\eta_{u}:=\nabla u \cdot \boldsymbol{p} .
$$

As we are only interested by situations in which the energy $E^{\varepsilon}\left(U^{\varepsilon}\right)+F^{\varepsilon}\left(U^{\varepsilon}, \theta^{\varepsilon}\right)$ remains bounded, $\varepsilon^{2} E^{\varepsilon}\left(U^{\varepsilon}\right)$ tends to zero and we get the constraint

$$
\bar{E}\left(v, \eta_{u}\right)=0 .
$$

This implies that, for any $\left(p, s, s^{\prime}\right) \in \mathcal{A}$,

$$
\left(v_{s^{\prime}}-v_{s}+\nabla u \cdot \boldsymbol{p}\right) \cdot \tau_{p, s, s^{\prime}}=0,
$$

from which we deduce that

$$
\left(\nabla v_{s^{\prime}} \cdot \boldsymbol{p}-\nabla v_{s} \cdot \boldsymbol{p}+\nabla \nabla u \cdot \boldsymbol{p} \cdot \boldsymbol{p}\right) \cdot \tau_{p, s, s^{\prime}}=0 .
$$

Using the two last equations, we get for any $\left(p, s, s^{\prime}\right) \in \mathcal{A}$

$$
\begin{array}{r}
\varepsilon^{-2}\left(U_{I+p, s^{\prime}}^{\varepsilon}-U_{I, s}^{\varepsilon}\right) \cdot \tau_{p, s, s^{\prime}}=\left(\frac{1}{2} \nabla \nabla u\left(y_{I}^{\varepsilon}\right) \cdot \boldsymbol{p} \cdot \boldsymbol{p}+\nabla v_{s^{\prime}}\left(y_{I}^{\varepsilon}\right) \cdot \boldsymbol{p}+\left(w_{s^{\prime}}\left(y_{I}^{\varepsilon}\right)-w_{s}\left(y_{I}^{\varepsilon}\right)\right)\right) \\
\cdot \tau_{p, s, s^{\prime}}+o(1)
\end{array}
$$

and thus

$$
\begin{aligned}
E^{\varepsilon}\left(U^{\varepsilon}\right) & =\sum_{I} \sum_{\left(p, s, s^{\prime}\right) \in \mathcal{A}} \frac{a_{p, s, s^{\prime}}}{2}\left(\frac{U_{I+p, s^{\prime}}^{\varepsilon}-U_{I, s}^{\varepsilon}}{\varepsilon^{2}} \cdot \tau_{p, s, s^{\prime}}\right)^{2} \\
& =\int_{\Omega} \sum_{\left(p, s, s^{\prime}\right) \in \mathcal{A}} \frac{a_{p, s, s^{\prime}}}{2}\left(\left(\frac{1}{2} \nabla \nabla u(x) \cdot \boldsymbol{p} \cdot \boldsymbol{p}+\nabla v_{s^{\prime}}(x) \cdot \boldsymbol{p}\right.\right. \\
& =\bar{E}\left(w, \xi_{u, v}\right)+o(1)
\end{aligned}
$$

where $\bar{E}$ is the functional defined in (11) and $\xi_{u, v}$ is the quantity

$$
\xi_{u, v}:=\frac{1}{2} \nabla \nabla u \cdot \boldsymbol{p} \cdot \boldsymbol{p}+\nabla v_{s^{\prime}} \cdot \boldsymbol{p} .
$$

On the other hand

$$
\begin{aligned}
\left(\alpha_{U}\right)_{I, p, s, s^{\prime}}^{\varepsilon}: & =\varepsilon^{-1} \tau_{p, s, s^{\prime}} \times\left(U_{I+p, s^{\prime}}^{\varepsilon}-U_{I, s}^{\varepsilon}\right) \\
& =\varepsilon^{-1} \tau_{p, s, s^{\prime}} \times\left(\left(u\left(y_{I+p}^{\varepsilon}\right)-u\left(y_{I}^{\varepsilon}\right)\right)+\varepsilon\left(v_{s^{\prime}}\left(y_{I+p}^{\varepsilon}\right)-v_{s}\left(y_{I}^{\varepsilon}\right)\right)+o(\varepsilon)\right) \\
& =\tau_{p, s, s^{\prime}} \times\left(\nabla u\left(y_{I}^{\varepsilon}\right) \cdot \boldsymbol{p}+v_{s^{\prime}}\left(y_{I}^{\varepsilon}\right)-v_{s}\left(y_{I}^{\varepsilon}\right)\right)+o(1) .
\end{aligned}
$$


Using also the fact that $\theta_{I+p, s^{\prime}}^{\varepsilon}=\theta\left(y_{I+p}^{\varepsilon}\right)=\theta\left(y_{I}^{\varepsilon}\right)+o(1)$ we get

$$
\begin{aligned}
& F^{\varepsilon}\left(U^{\varepsilon}, \theta^{\varepsilon}\right):= \\
& \sum_{I} \sum_{\left(p, s, s^{\prime}\right)}\left[\left(\theta_{I, s}^{\varepsilon}-\left(\alpha_{U}\right)_{I, p, s, s^{\prime}}^{\varepsilon}\right) \cdot \frac{B_{p, s, s^{\prime}}}{2} \cdot\left(\theta_{I, s}^{\varepsilon}-\left(\alpha_{U}\right)_{I, p, s, s^{\prime}}^{\varepsilon}\right)\right. \\
& \quad+\left(\theta_{I, s}^{\varepsilon}-\left(\alpha_{U}\right)_{I, p, s, s^{\prime}}^{\varepsilon}\right) \cdot C_{p, s, s^{\prime}} \cdot\left(\theta_{I+p, s^{\prime}}^{\varepsilon}-\left(\alpha_{U}\right)_{I, p, s, s^{\prime}}^{\varepsilon}\right) \\
& \left.\quad+\left(\theta_{I+p, s^{\prime}}^{\varepsilon}-\left(\alpha_{U}\right)_{I, p, s, s^{\prime}}^{\varepsilon}\right) \cdot \frac{D_{p, s, s^{\prime}}}{2} \cdot\left(\theta_{I+p, s^{\prime}}^{\varepsilon}-\left(\alpha_{U}\right)_{I, p, s, s^{\prime}}^{\varepsilon}\right)\right] \\
& =\bar{F}\left(v, \eta_{u}, \theta\right)+o(1),
\end{aligned}
$$

where we define

$$
\begin{aligned}
\bar{F}(v, \eta, \theta):= & \int_{\Omega} \sum_{p, s, s^{\prime}}\left[\left(\theta_{s}(x)-\frac{\tau_{p, s, s^{\prime}}}{\ell_{p, s, s^{\prime}}} \times\left(v_{s^{\prime}}(x)-v_{s}(x)+\eta_{p, s^{\prime}}(x)\right)\right)\right. \\
& \cdot \frac{B_{p, s, s^{\prime}}}{2} \cdot\left(\theta_{s}(x)-\frac{\tau_{p, s, s^{\prime}}}{\ell_{p, s, s^{\prime}}} \times\left(v_{s^{\prime}}(x)-v_{s}(x)+\eta_{p, s^{\prime}}(x)\right)\right) \\
+ & \left(\theta_{s}(x)-\frac{\tau_{p, s, s^{\prime}}}{\ell_{p, s, s^{\prime}}} \times\left(v_{s^{\prime}}(x)-v_{s}(x)+\eta_{p, s^{\prime}}(x)\right)\right) \\
& \cdot C_{p, s, s^{\prime}} \cdot\left(\theta_{s^{\prime}}(x)-\frac{\tau_{p, s, s^{\prime}}}{\ell_{p, s, s^{\prime}}} \times\left(v_{s^{\prime}}(x)-v_{s}(x)+\eta_{p, s^{\prime}}(x)\right)\right) \\
+ & \left(\theta_{s^{\prime}}(x)-\frac{\tau_{p, s, s^{\prime}}}{\ell_{p, s, s^{\prime}}} \times\left(v_{s^{\prime}}(x)-v_{s}(x)+\eta_{p, s^{\prime}}(x)\right)\right) \\
& \left.\cdot \frac{D_{p, s, s^{\prime}}}{2} \cdot\left(\theta_{s^{\prime}}(x)-\frac{\tau_{p, s, s^{\prime}}}{\ell_{p, s, s^{\prime}}} \times\left(v_{s^{\prime}}(x)-v_{s}(x)+\eta_{p, s^{\prime}}(x)\right)\right)\right] .
\end{aligned}
$$

To summarize, the effective energy is $\bar{E}\left(w, \xi_{u, v}\right)+\bar{F}\left(v, \eta_{u}, \theta\right)$ under the constraint $\bar{E}\left(v, \eta_{u}\right)=0$. As $w$ is an internal variable and as, in general, we also have no external action on $\theta$, it is better to write the effective energy in terms of the macroscopic displacement $u$ only:

Theorem 1. The limit (effective) energy associated with the microscopic energy $E^{\varepsilon}+F^{\varepsilon}$ is

$$
\mathscr{E}(u):=\inf _{w, v, \theta}\left\{\bar{E}\left(w, \xi_{u, v}\right)+\bar{F}\left(v, \eta_{u}, \theta\right): \bar{E}\left(v, \eta_{u}\right)=0\right\}
$$

We remark that the constraint $\bar{E}\left(v, \eta_{u}\right)=0$ may induce a constraint on the strain tensor $e(u)$ (i.e., the symmetric part of $\nabla u$ ). Indeed we will see that the effective behavior of the considered structure is often subject to some constraints (like inextensibility in some direction, incompressibility, or even total rigidity). 
We also expect that the effective energy corresponds to a strain gradient model. Indeed the second gradient of $u$ enters the expression of $\bar{E}\left(w, \xi_{u, v}\right)$ directly through the definition (14) of $\xi_{u, v}$. Moreover, the constraint $\bar{E}\left(v, \eta_{u}\right)=0$ establishes a linear relation between $v$ and $\nabla u$; thus, the dependence of $\xi_{u, v}$ on the gradient of $v$ can be a second source for strain gradient terms. However, it is not so simple to find structures for which such strain gradient effects arise and are not concealed by the constraint. In the next section we explain how to compute explicitly the limit energy, and we apply this procedure to many examples in Section 5.

Note that we prefer to describe the homogenized behavior of the considered structures in terms of the limit elastic energy only. Beyond the fact that it is very concise, it has the advantage of being written without considering any applied external forces. Indeed external forces have little to do with the constitutive law of the new material. Equilibrium equations under the action of a (reasonable) external force field $f$ can then be obtained by simply writing the Euler equations of the minimization of the total energy $\mathscr{E}(u)-\int_{\Omega} f(x) \cdot u(x) d x$. Properties of $\Gamma$-convergence [Braides 2002; Dal Maso 1993] ensure that the equilibrium states of the considered structure converge towards this minimum.

\section{Explicit computation of the homogenized stiffness matrices}

Let us describe the algorithm which makes explicit the limit energy. We give here all the details needed for understanding the Octave/MATLAB software we provide in [Abdoul-Anziz and Seppecher 2017].

Note first that in the computations leading to Theorem 1, we have assumed $|Y|=1$. As it is sometimes clearer to describe the structure using a prototype cell which does not satisfy $|Y|=1$, all geometric quantities have to be rescaled (i.e., divided by $|Y|^{1 / N}$ ) before using the following algorithm.

Both limit energies $\bar{E}\left(v, \eta_{u}\right)$ and $\bar{F}\left(v, \eta_{u}, \theta\right)$ are quadratic forms of their variables. A priori the variables $v$ and $\theta$ are $K \times 3$ matrices $v_{s, i}$ and $\theta_{s, i}$ with $s \in$ $\{1, \ldots, K\}$ and $i \in\{1,2,3\}$, while the variable $\eta$ is an $n \times K \times 3$ tensor $\eta_{p, s, i}$ with $p \in \mathcal{P}$. From now on we identify them with $3 K$ or $3 n K$ vectors $v_{(s, i)}, \theta_{(s, i)}$, and $\eta_{(p, s, i)}$ without modifying the notation. In the same way $(\nabla u)_{i, \gamma}$ and $(\nabla \nabla u)_{i, \gamma, \gamma^{\prime}}$ are identified with the $3 N$ and $3 N^{2}$ vectors $(\nabla u)_{(i, \gamma)}$ and $(\nabla \nabla u)_{\left(i, \gamma, \gamma^{\prime}\right)}$ without modifying the notation.

Step 1 (rewriting the energies (11) and (15) in canonical form).

$$
\begin{aligned}
\bar{E}(v, \eta) & =\frac{1}{2} \int_{\Omega} v^{t} \cdot \boldsymbol{A} \cdot v+\eta^{t} \cdot \boldsymbol{B} \cdot \eta+2 v^{t} \cdot \boldsymbol{C} \cdot \eta \\
\bar{F}(v, \eta, \theta) & =\frac{1}{2} \int_{\Omega} v^{t} \cdot \boldsymbol{D} \cdot v+\eta^{t} \cdot \boldsymbol{E} \cdot \eta+\theta^{t} \cdot \boldsymbol{F} \cdot \theta+2 v^{t} \cdot \boldsymbol{G} \cdot \eta+2 \theta^{t} \cdot \boldsymbol{H} \cdot v+2 \theta^{t} \cdot \boldsymbol{J} \cdot \eta .
\end{aligned}
$$


This is a simple assembly process. Indeed, denoting $\tilde{a}_{p, s, r, i, j}:=a_{p, s, r} \tau_{p, s, r, i} \tau_{p, s, r, j}$,

$$
\begin{aligned}
& \left\{\begin{array}{l}
\boldsymbol{A}_{(s, i),(r, j)}:=-\sum_{p \in \mathcal{P}}\left(\tilde{a}_{p, s, r, i, j}+\tilde{a}_{p, r, s, i, j}\right) \quad \text { if } r \neq s, \\
\boldsymbol{A}_{(s, i),(s, j)}:=-\sum_{r \neq s} \boldsymbol{A}_{(s, i),(r, j)},
\end{array}\right. \\
& \left\{\begin{array}{l}
\boldsymbol{B}_{(p, s, i),(q, r, j)}:=0 \quad \text { if }(q, r) \neq(p, s), \\
\boldsymbol{B}_{(p, s, i),(p, s, j)}:=\sum_{r} \tilde{a}_{p, r, s, i, j} .
\end{array}\right. \\
& \left\{\begin{array}{l}
\boldsymbol{C}_{(s, i),(p, r, j)}:=-\tilde{a}_{p, s, r, i, j} \\
\boldsymbol{C}_{(s, i),(p, s, j)}:=-\sum_{r \neq s} \boldsymbol{C}_{(s, i),(p, r, j)},
\end{array}\right.
\end{aligned}
$$

and using the Levi-Civita symbol $\epsilon$ and denoting

$$
\tilde{b}_{p, s, r, i, j}:=\sum_{k, k^{\prime}, l, l^{\prime}} \frac{1}{\ell_{p, s, r}^{2}} \epsilon_{i, k, l} \epsilon_{j, k^{\prime}, l^{\prime}}(b+2 c+d)_{p, s, r, k, k^{\prime}} \tau_{p, s, r, l} \tau_{p, s, r, l^{\prime}},
$$

we have

$$
\begin{aligned}
& \left\{\begin{array}{l}
\boldsymbol{D}_{(s, i),(r, j)}:=-\sum_{p \in \mathcal{P}}\left(\tilde{b}_{p, s, r, i, j}+\tilde{b}_{p, r, s, i, j}\right) \quad \text { if } r \neq s, \\
\boldsymbol{D}_{(s, i),(s, j)}:=-\sum_{r \neq s} \boldsymbol{D}_{(s, i),(r, j)},
\end{array}\right. \\
& \left\{\begin{array}{l}
\boldsymbol{E}_{(p, s, i),(q, r, j)}:=0 \quad \text { if }(q, r) \neq(p, s), \\
\boldsymbol{E}_{(p, s, i),(p, s, j)}:=\sum_{r} \tilde{b}_{p, r, s, i, j} .
\end{array}\right. \\
& \left\{\begin{array}{l}
\boldsymbol{G}_{(s, i),(p, r, j)}:=-\tilde{b}_{p, s, r, i, j} \quad \text { if } r \neq s, \\
\boldsymbol{G}_{(s, i),(p, s, j)}:=-\sum_{r \neq s} \boldsymbol{G}_{(s, i),(p, r, j)},
\end{array}\right. \\
& \left\{\begin{array}{l}
\boldsymbol{F}_{(s, i),(r, j)}:=2 \sum_{p \in \mathcal{P}} c_{p, s, r, i, j} \\
\boldsymbol{F}_{(s, i),(s, j)}:=\sum_{p \in \mathcal{P}}\left(2 c_{p, s, s, i, j}+\sum_{r}\left(b_{p, s, r, i, j}+d_{p, r, s, i, j}\right)\right), \\
\boldsymbol{H}_{(s, i),(s, j)}:=-\sum_{r \neq s} \boldsymbol{H}_{(s, i),(r, j)},
\end{array}\right. \\
& \left\{\begin{array}{l}
\boldsymbol{J}_{(s, i),(p, r, j)}:=\sum_{p \in \mathcal{P}} \sum_{k, l}\left(1 / \ell_{p, s, r}\right) \epsilon_{j, l, k}(b+c)_{p, s, r, l, i} \tau_{p, s, r, k} \\
\boldsymbol{J}_{(s, i),(p, s, j)}:=\sum_{p \in \mathcal{P}} \sum_{k, l}\left(1 / \ell_{p, s, s}\right) \epsilon_{j, l, k}\left((b+c)_{p, s, s, l, i}\right. \\
\left.\quad \text { if } r \neq \sum_{r \neq s}(c+d)_{p, s, r, l, i}\right) \tau_{p, s, r, k} .
\end{array}\right.
\end{aligned}
$$

Step 2 (computing the constraint). Using the canonical form it is easy to compute the minimum of $\bar{E}\left(v, \eta_{u}\right)$ with respect to $v$. When the minimum is attained, $v$ satisfies $\boldsymbol{A} \cdot v+\boldsymbol{C} \cdot \eta_{u}=0$. The vector $\bar{v}:=-\boldsymbol{A}^{+} \cdot \boldsymbol{C} \cdot \eta_{u}$ where $\boldsymbol{A}^{+}$stands for the pseudoinverse of $\boldsymbol{A}$ is a possible solution ${ }^{5}$ and the minimal value is $\int_{\Omega} \frac{1}{2} \eta_{u}^{t} \cdot \boldsymbol{X} \cdot \eta_{u}$ where

$$
\boldsymbol{X}:=\boldsymbol{B}-\boldsymbol{C}^{t} \cdot \boldsymbol{A}^{+} \cdot \boldsymbol{C} .
$$

Note that the minimum with respect to $w$ of $\bar{E}\left(w, \xi_{u, v}\right)$ is computed in a similar way and becomes $\int_{\Omega} \frac{1}{2}\left(\xi_{u, v}\right)^{t} \cdot \boldsymbol{X} \cdot \xi_{u, v}$.

${ }^{5}$ The properties of the Moore-Penrose pseudoinverse imply that this vector $\bar{v}$ belongs to the orthogonal to the kernel of $A$ and so satisfies $\sum_{k} v_{k}=0$. 
Let us now introduce a linear operator $\boldsymbol{L}$ by setting, for any $p \in \mathcal{P}, s \in\{1, \ldots, K\}$, $1 \leq i, j \leq 3$, and $1 \leq \gamma \leq n$,

$$
\boldsymbol{L}_{(p, s, i),(j, \gamma)}:=\delta_{i, j}\left(\boldsymbol{p} \cdot t_{\gamma}\right),
$$

where $\delta$ stands for the Kronecker delta so that

$$
\eta_{u}=\boldsymbol{L} \cdot \nabla u
$$

Setting $\boldsymbol{Q}:=\boldsymbol{L}^{t} \cdot \boldsymbol{X} \cdot \boldsymbol{L}$ and $\boldsymbol{K}:=-\boldsymbol{A}^{+} \cdot \boldsymbol{C} \cdot \boldsymbol{L}$, the constraint $\bar{E}\left(v, \eta_{u}\right)=0$ reads

$$
\begin{aligned}
\boldsymbol{Q} \cdot \nabla u & =0, \\
v & =\boldsymbol{K} \cdot \nabla u+\tilde{v} \quad \text { with } \tilde{v} \in \operatorname{Ker}(\boldsymbol{A}) .
\end{aligned}
$$

Note that the matrix $\boldsymbol{Q}$ would have been the homogenized stiffness matrix of our structure if we had assumed a less stiff behavior of the interactions: we recover here results which have been obtained recently by [Martinsson and Babuška 2007b]. As we are, on the contrary, interested here in structures made by a very stiff material, we have to focus only on the kernel of $\boldsymbol{Q}$. Objectivity implies that it contains at least the skew-symmetric matrices, ${ }^{6}$ but in the most interesting cases it is much larger.

We introduce an orthonormal basis $\left(W^{\xi}\right)_{\xi=1}^{d}$ of $\operatorname{Ker}(\boldsymbol{Q})(N(5-N) / 2 \leq d \leq 3 N)$. The matrix $\boldsymbol{P}_{(i, \gamma),\left(j, \gamma^{\prime}\right)}:=\sum_{\xi} W_{(i, \gamma)}^{\xi} W_{\left(j, \gamma^{\prime}\right)}^{\xi}$ represents the projection onto $\operatorname{Ker}(\boldsymbol{Q})$, and constraint (20) reads

$$
\nabla u=\boldsymbol{P} \cdot \nabla u
$$

On the other hand, in order to represent $\tilde{v}$, we introduce a basis $\left(V^{\xi}\right)_{\xi=1}^{\tilde{d}}$ of $\operatorname{Ker}(\boldsymbol{A})(3 \leq \tilde{d} \leq 3 K)$ : we set $\tilde{v}=\sum_{\xi=1}^{d} b_{\xi}(x) V^{\xi}$, that is, $\tilde{v}=\boldsymbol{V} \cdot b$ with $\boldsymbol{V}_{(s, i), \xi}:=$ $V_{(s, i)}^{\xi}$.

Remark. Surprisingly enough, the variable $b$ which is introduced at this step may play an important role. It represents $\tilde{v}$ that is internal degrees of freedom (free only with respect to the highest-order energy). In classical homogenization $\tilde{v}$ is constant in the unit cell $Y$, and thus, its value has no effect on the homogenized energy. That is why one usually fixes, without loss of generality, the mean value of $v$ to be zero. Here the situation is completely different: due to high contrast and for special geometries, the set in which $\tilde{v}$ lives may be much richer and the effect of this extra kinematic variable on the homogenized energy may be fundamental. This fact is illustrated by some examples in Section 5 .

\footnotetext{
${ }^{6}$ By saying that a $3 \times N$ matrix $M$ is skew-symmetric we mean that, for all $1 \leq \gamma, \gamma^{\prime} \leq N$, $M_{\gamma, \gamma^{\prime}}+M_{\gamma^{\prime}, \gamma}=0$.
} 
Step 3 (computing the extensional part of the energy). Owing to (20), we have $(\nabla v)_{s, i, \gamma}=\sum_{j, \gamma^{\prime}}\left(K_{(s, i),\left(j, \gamma^{\prime}\right)}(\nabla \nabla u)_{j, \gamma^{\prime}, \gamma}\right)+(\nabla \tilde{v})_{s, i, \gamma}$. Using (22) and expressing $\tilde{v}$ in the base $\left(V^{\xi}\right)$, we can rewrite (14) as

$$
\xi_{u, v}=\boldsymbol{M} \cdot \nabla \nabla u+\boldsymbol{N} \cdot \nabla b
$$

with

$$
\begin{aligned}
\boldsymbol{M}_{(p, s, i),\left(j, \gamma, \gamma^{\prime}\right)} & =\sum_{\zeta} \sum_{k}\left(\boldsymbol{K}_{(s, i),(k, \zeta)}+\frac{1}{2} \boldsymbol{p}_{\zeta} \delta_{i, k}\right) \boldsymbol{P}_{(k, \zeta),(j, \gamma)} \boldsymbol{p}_{\gamma^{\prime}}, \\
\boldsymbol{N}_{(p, s, i),(\xi, \gamma)} & =V_{(s, i)}^{\xi} \boldsymbol{p}_{\gamma} .
\end{aligned}
$$

The extensional energy $\inf _{w} \bar{E}\left(w, \xi_{u, v}\right)=\frac{1}{2} \int_{\Omega}\left(\xi_{u, v}\right)^{t} \cdot \boldsymbol{X} \cdot \xi_{u, v}$ becomes

$\frac{1}{2} \int_{\Omega}(\nabla \nabla u)^{t} \cdot \boldsymbol{M}^{t} \cdot \boldsymbol{X} \cdot \boldsymbol{M} \cdot \nabla \nabla u+(\nabla b)^{t} \cdot \boldsymbol{N}^{t} \cdot \boldsymbol{X} \cdot \boldsymbol{N} \cdot \nabla b+2(\nabla \nabla u)^{t} \cdot \boldsymbol{M}^{t} \cdot \boldsymbol{X} \cdot \boldsymbol{N} \cdot \nabla b$.

We prefer to rewrite it as the sum of two nonnegative terms:

$$
\inf _{w} \bar{E}\left(w, \xi_{u, v}\right)=\frac{1}{2} \int_{\Omega}(\nabla \nabla u)^{t} \cdot \mathcal{R} \cdot \nabla \nabla u+(\nabla b+\mathcal{T} \cdot \nabla \nabla u)^{t} \cdot \mathcal{S} \cdot(\nabla b+\mathcal{T} \cdot \nabla \nabla u)
$$

where

$\mathcal{S}:=\boldsymbol{N}^{t} \cdot \boldsymbol{X} \cdot \boldsymbol{N}, \quad \mathcal{T}:=\mathcal{S}^{+} \cdot \boldsymbol{N}^{t} \cdot \boldsymbol{X} \cdot \boldsymbol{M}, \quad$ and $\quad \mathcal{R}:=\boldsymbol{M}^{t} \cdot \boldsymbol{X} \cdot \boldsymbol{M}-\boldsymbol{M}^{t} \cdot \boldsymbol{X} \cdot \boldsymbol{N} \cdot \mathcal{T}$.

Step 4 (computing the flexural part of the energy). We can also easily compute the minimum with respect to $\theta$ of $\bar{F}\left(v, \eta_{u}, \theta\right)$. When the minimum is attained, $\theta$ satisfies $\boldsymbol{F} \cdot \theta+\boldsymbol{H} \cdot v+\boldsymbol{J} \cdot \eta_{u}=0$. The vector $\bar{\theta}:=-\boldsymbol{F}^{+} \cdot\left(\boldsymbol{H} \cdot v+\boldsymbol{J} \cdot \eta_{u}\right)$ is a possible solution, and the minimal value is

$$
\begin{aligned}
\inf _{\theta} \bar{F}\left(v, \eta_{u}, \theta\right)=\frac{1}{2} \int_{\Omega} v^{t} \cdot\left(\boldsymbol{D}-\boldsymbol{H}^{t} \cdot \boldsymbol{F}^{+} \cdot \boldsymbol{H}\right) \cdot v+ & \eta_{u}^{t} \cdot\left(\boldsymbol{E}-\boldsymbol{J}^{t} \cdot \boldsymbol{F}^{+} \cdot \boldsymbol{J}\right) \cdot \eta_{u} \\
& +2 v^{t} \cdot\left(\boldsymbol{G}-\boldsymbol{H}^{t} \cdot \boldsymbol{F}^{+} \cdot \boldsymbol{J}\right) \cdot \eta_{u} .
\end{aligned}
$$

Using (22), let us replace $\eta_{u}$ by $\boldsymbol{L} \cdot \nabla u=\boldsymbol{L} \cdot \boldsymbol{P} \cdot \nabla u$ and $v$ by $\boldsymbol{K} \cdot \boldsymbol{P} \cdot \nabla u+\boldsymbol{V} \cdot b$. We get

$$
\inf _{\theta} \bar{F}\left(v, \eta_{u}, \theta\right)=\frac{1}{2} \int_{\Omega} b^{t} \cdot S \cdot b+(\nabla u)^{t} \cdot \boldsymbol{Z} \cdot \nabla u+2 b^{t} \cdot \boldsymbol{Y} \cdot \nabla u
$$

with

$$
\begin{aligned}
\boldsymbol{S}:=\boldsymbol{V}^{t} \cdot\left(\boldsymbol{D}-\boldsymbol{H}^{t} \cdot \boldsymbol{F}^{+} \cdot \boldsymbol{H}\right) \cdot \boldsymbol{V}, \\
\boldsymbol{Z}:=\boldsymbol{P}^{t} \cdot\left(\boldsymbol{K}^{t} \cdot\left(\boldsymbol{D}-\boldsymbol{H}^{t} \cdot \boldsymbol{F}^{+} \cdot \boldsymbol{H}\right) \cdot \boldsymbol{K}+\boldsymbol{L}^{t} \cdot\left(\boldsymbol{E}-\boldsymbol{J}^{t} \cdot \boldsymbol{F}^{+} \cdot \boldsymbol{J}\right) \cdot \boldsymbol{L}\right. \\
\left.\quad+\boldsymbol{K}^{t} \cdot\left(\boldsymbol{G}-\boldsymbol{H}^{t} \cdot \boldsymbol{F}^{+} \cdot \boldsymbol{J}\right) \cdot \boldsymbol{L}+\boldsymbol{L}^{t} \cdot\left(\boldsymbol{G}^{t}-\boldsymbol{J}^{t} \cdot \boldsymbol{F}^{+} \cdot \boldsymbol{K}\right) \cdot L\right) \cdot \boldsymbol{P}, \\
\quad \begin{array}{r}
\boldsymbol{Y}:=\boldsymbol{V}^{t} \cdot\left(\left(\boldsymbol{D}-\boldsymbol{H}^{t} \cdot \boldsymbol{F}^{+} \cdot \boldsymbol{H}\right) \cdot \boldsymbol{K}+\left(\boldsymbol{G}-\boldsymbol{H}^{t} \cdot \boldsymbol{F}^{+} \cdot \boldsymbol{J}\right) \cdot \boldsymbol{L}\right) \cdot \boldsymbol{P} .
\end{array}
\end{aligned}
$$


Again we prefer to write this energy as the sum of two nonnegative terms:

$$
\inf _{\theta} \bar{F}\left(v, \eta_{u}, \theta\right)=\frac{1}{2} \int_{\Omega}(\nabla u)^{t} \cdot \boldsymbol{R} \cdot \nabla u+(b+\boldsymbol{T} \cdot \nabla u)^{t} \cdot \boldsymbol{S} \cdot(b+\boldsymbol{T} \cdot \nabla u)
$$

with

$$
\boldsymbol{T}:=\boldsymbol{S}^{+} \cdot \boldsymbol{Y} \quad \text { and } \quad \boldsymbol{R}:=\boldsymbol{Z}-\boldsymbol{Y}^{t} \cdot \boldsymbol{T} .
$$

Collecting the results. The limit energy $\mathcal{E}$ obtained by collecting (23) and (24), namely

$$
\begin{array}{r}
\mathcal{E}=\inf _{b} \frac{1}{2} \int_{\Omega}(\nabla \nabla u)^{t} \cdot \mathcal{R} \cdot \nabla \nabla u+(\nabla b+\mathcal{T} \cdot \nabla \nabla u)^{t} \cdot \mathcal{S} \cdot(\nabla b+\mathcal{T} \cdot \nabla \nabla u) \\
+(\nabla u)^{t} \cdot \boldsymbol{R} \cdot \nabla u+(b+\boldsymbol{T} \cdot \nabla u)^{t} \cdot \boldsymbol{S} \cdot(b+\boldsymbol{T} \cdot \nabla u),
\end{array}
$$

appears to be the integral of a quadratic form depending on the first and second gradients of the macroscopic displacement and on an extra kinematic variable $b$ and its first gradient. The limit model is both a second gradient model (or strain gradient model) and a generalized continuum. From now on, we will call "microadjustment" the variable $b$.

In general we cannot go further because the microadjustment cannot be computed locally. The equilibrium equations are a coupled linear system of partial differential equations for $u$ and $b$. This system is fixed as soon as the matrices $\boldsymbol{Q}$, $\mathcal{R}, \mathcal{S}, \mathcal{T}, \boldsymbol{R}, \boldsymbol{S}$, and $\boldsymbol{T}$ are fixed.

Step 5 (when possible, eliminating the extra kinematic variable). However, it is sometimes still possible to eliminate the microadjustment. That is the case when, for any field $u$, there exists a field $\bar{b}$ such that

$$
\mathcal{S} \cdot(\nabla \bar{b}+\mathcal{T} \cdot \nabla \nabla u)=0 \quad \text { and } \quad \boldsymbol{S} \cdot(\bar{b}+\boldsymbol{T} \cdot \nabla u)=0 .
$$

Note that this operation would lead to serious difficulties if nonfree boundary conditions were considered. This field $\bar{b}$ clearly minimizes the energy and the homogenized energy reduces to

$\mathscr{E}(u)=\frac{1}{2} \int_{\Omega}(\nabla \nabla u)^{t} \cdot \mathcal{R} \cdot \nabla \nabla u+(\nabla u)^{t} \cdot \boldsymbol{R} \cdot \nabla u \quad$ under the constraint $\quad \boldsymbol{Q} \cdot \nabla u=0$.

Let us emphasize that the cases where the microadjustment $b$ cannot be eliminated are also very relevant: we will see in the examples given in Section 5 that we recover many models of generalized continua which are widely used by mechanicians in practical situations.

Implementation. The algorithm we just described for determining the homogenized energy (25) or (27) is pure linear algebra dealing with very low-dimension matrices. It is very easy to implement in languages like Octave or MATLAB (an Octave/MATLAB package can be found in [Abdoul-Anziz and Seppecher 2017]) 
for getting numerical results or like Maxima for obtaining analytical results. As no optimization is needed, it can even be implemented in JavaScript (an online JavaScript tool is in development).

However, two points are not automated. Before using the algorithm, one has to manually check that the connectedness condition is satisfied. Then for using (27) one also has to check that equations (26) admit a solution; otherwise, one has to deal with the generalized continuum model given by (25).

In view of our results, the effective energy may correspond to a second gradient model (i.e., a strain gradient model) possibly coupled with an extra kinematic variable and subject to some first gradient constraints. However, few periodic structures exhibit such a behavior. Indeed most of them present a nondegenerate energy $\bar{E}$; in that case the strong constraint $\boldsymbol{Q} \cdot \nabla u=0$ hides any second gradient effect. But even when $\boldsymbol{Q}$ is degenerate, it happens frequently that $\mathcal{R}=0$ : the model remains degenerate after rescaling. Different cases will be illustrated in the next section. We have no way, other than the algorithm we just described, for predicting whether a limit energy model will correspond to a classical model or a second gradient one or even a generalized continuum.

\section{Examples}

We apply the procedure described in the previous section to different $2 \mathrm{D}$ or $3 \mathrm{D}$ examples following the cases described in the introduction. To fix the ideas we always choose $a_{p, s, r}=1$ whenever two nodes are interacting (i.e., when $a_{p, s, r} \neq 0$ ). Note that this assumption means that the sections of the bars differ when their lengths differ. We also always choose $f=1$ (and, in the 3D case, $t=0.25$ ). We classify our examples by the dimension $N=1,2,3$ of the space in which the homogenized energy lives, leading thus to beams, membranes or plates, or 3D materials. In the cases $N=1$ or $N=2$ we successively consider 2D and 3D examples. We write the effective energy in terms of the components $e_{i, j}(u)$ of the strain tensor $e(u)$ and of the components $\partial^{2} u_{i} / \partial x_{j} \partial x_{k}$ of the second gradient of the displacement. Translating the results in terms of the strain gradient is straightforward. Mind that the presence of constraints allows us to write different forms for the expression of the limit energy.

5.1. Beams. For sake of simplicity let us start by considering structures in $\mathbb{R}^{2}$ with one vector of periodicity $(N=1)$.

5.1.1. $2 D$ Warren beam. We consider the geometry (see Figure 3 ) $\Omega=(0,1)$, $K=2, y_{1}=(0,0), y_{2}=(0,1), t_{1}=(1,0)$, and $a_{1,1,2}=a_{2,1,1}=a_{2,2,2}=a_{2,1,2}=1$; all other components $a_{p, s, s^{\prime}}$ vanish. For this well known structure, the constraint (22) reads $e_{1,1}(u)=0$ : the beam is inextensible. A simple solution for condition (26) 


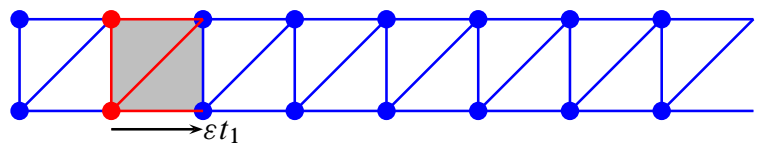

Figure 3. Warren beam.

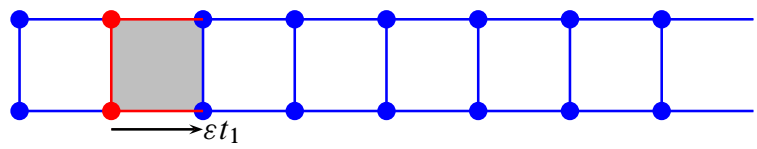

Figure 4. Square periodic beam.

is $b=0$ and the limit energy reads

$$
\mathscr{E}(u)=\frac{1}{2} \int_{0}^{1} \lambda\left(\frac{\partial^{2} u_{2}}{\partial x_{1}^{2}}\right)^{2} d x_{1}
$$

(with $\lambda=\frac{1}{2}$ ) and thus corresponds to an inextensible Euler-Bernoulli beam model.

Remark. The inextensibility constraint deserves a comment. This constraint is due to the order of magnitude we have assumed for the stiffness of the material the structure is made of. We already noticed that this assumption is actually an assumption for the order of magnitude of the forces which act on the structure. In other words we have implicitly assumed that the applied forces are not strong enough for extending the beam but that they are strong enough for bending it. Of course, different assumptions corresponding to different practical situations could be considered. The constraints which arise in all the following examples must be interpreted in this way.

5.1.2. Square periodic beam. The geometry is similar to the previous example (see Figure 4). We simply delete the diagonal bars by setting $a_{2,1,2}=0$. The constraint (22) still reads $e_{1,1}(u)=0$, but the homogenized energy now reads

$$
\mathscr{E}(u)=\inf _{b} \frac{1}{2} \int_{0}^{1}\left(\left(\frac{\partial b_{2}}{\partial x_{1}}\right)^{2}+\left(\frac{\partial b_{1}}{\partial x_{1}}\right)^{2}+2\left(-b_{1}+b_{2}+\frac{\partial u_{2}}{\partial x_{1}}\right)^{2}\right) d x_{1}
$$

Denoting $\varphi:=b_{1}-b_{2}$ and minimizing with respect to $b_{1}+b_{2}$ (by choosing $b_{1}+b_{2}=$ $0)$, the limit energy becomes

$$
\mathscr{E}(u)=\inf _{\varphi} \frac{1}{2} \int_{0}^{1}\left(\lambda\left(\frac{\partial \varphi}{\partial x_{1}}\right)^{2}+\zeta\left(\frac{\partial u_{2}}{\partial x_{1}}-\varphi\right)^{2}\right) d x_{1}
$$

(with $\lambda=\frac{1}{2}$ and $\zeta=2$ ) and thus corresponds to an inextensible Timoshenko beam model. It is well known that this model is nonlocal (in terms of $u$ only) and that 


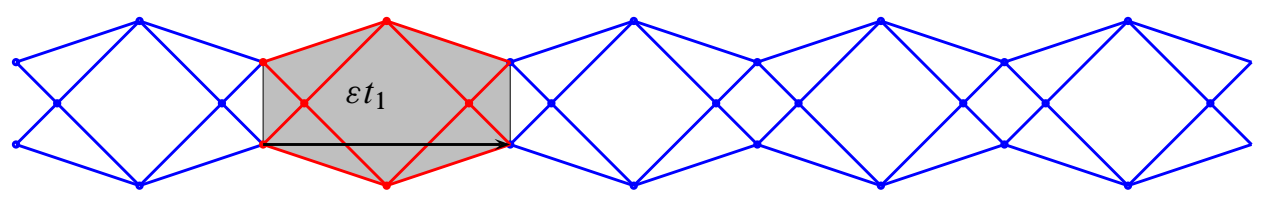

Figure 5. Pantographic beam $P_{e_{1}, e_{2}}$.

the extra kinematic variable $\varphi$ cannot be locally eliminated. The remaining part of our "microadjustment" coincides with the Timoshenko extra variable usually interpreted as the "rotation of the section".

5.1.3. Pantographic beam. Structures based on a pantograph have been the first (and almost the only ones) to give a microscopic interpretation to the propagation of dilatation, a characteristic feature of complete second gradient models. The structures have been studied using formal homogenization techniques [Rahali et al. 2015; Seppecher et al. 2011; Madeo et al. 2017], $\Gamma$-convergence tools [Alibert et al. 2003; Alibert and Della Corte 2015], numerical simulations [Giorgio 2016; Turco et al. 2016a], and even experiments [dell'Isola et al. 2016a; Turco et al. 2016b]. Our procedure makes their study easy.

We consider a planar beam with a cell made of six nodes $y_{1}=\frac{1}{6} e_{2}, y_{2}=-\frac{1}{6} e_{2}$, $y_{3}=\frac{1}{6} e_{1}, y_{4}=\frac{1}{6}\left(3 e_{1}+2 e_{2}\right), y_{5}=\frac{1}{6}\left(3 e_{1}-2 e_{2}\right)$, and $y_{6}=\frac{5}{6} e_{1} ;$ a periodicity vector $t_{1}=e_{1}$; and $a_{1,1,3}=a_{1,1,4}=a_{1,2,3}=a_{1,2,5}=a_{1,3,4}=a_{1,3,5}=a_{1,4,6}=a_{1,5,6}=$ $a_{2,4,1}=a_{2,5,2}=a_{2,6,1}=a_{2,6,2}=1$; all other components of the matrices $a_{1}$ and $a_{2}$ vanish.

This beam (see Figure 5) which lies along the line $\left(0, e_{1}\right)$ and belongs to the plane $\left(e_{1}, e_{2}\right)$ is denoted $P_{e_{1}, e_{2}}$ for further purpose.

In that case (22) gives no constraint. A possible solution for condition (26) is $b=(4,0,-1,-1,1,1) / 4 \times \frac{\partial u_{3}}{\partial x_{1}}$. The limit energy reads

$$
\mathscr{E}(u)=\frac{1}{2} \int_{0}^{1}\left(\lambda\left(\frac{\partial^{2} u_{1}}{\partial x_{1}^{2}}\right)^{2}+\mu\left(\frac{\partial^{2} u_{2}}{\partial x_{1}^{2}}\right)^{2}+\zeta\left(\frac{\partial u_{1}}{\partial x_{1}}\right)^{2}\right) d x_{1}
$$

(with $\lambda=\frac{2}{23}, \mu=\frac{2}{63}$, and $\zeta=324$ ). We recover the results obtained in [Seppecher et al. 2011; Alibert et al. 2003; Alibert and Della Corte 2015] where the exotic properties of this pantographic structure have been detailed. Its main specificity lies in the fact that a dilatation imposed in a part of the beam tends to spread on the whole beam. This phenomenon is due to the term $\left(\frac{\partial^{2} u_{1}}{\partial x_{1}^{2}}\right)^{2}$ and damped by the term $\left(\frac{\partial u_{1}}{\partial x_{1}}\right)^{2}$. This competition endows the model with intrinsic length $\sqrt{\lambda / \zeta}$.

5.1.4. $3 D$ Warren beam. The previous examples deal with planar beams. In that case the energy is, of course, degenerate with respect to out-of-plane displacements. Let us give a single example of a Warren-type 3D beam leading to an 


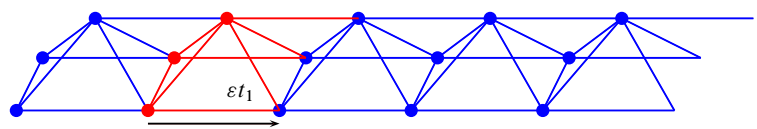

Figure 6. 3D Warren beam.

Euler-Bernoulli beam. The geometry (see Figure 6) is now $\Omega=(0,1), K=3$, $y_{1}=\left(0,0,-\frac{1}{2}\right), y_{2}=\left(0,0, \frac{1}{2}\right), y_{3}=\left(\frac{1}{2}, 0, \sqrt{2} / 2\right), t_{1}=(1,0)$, and $a_{1,1,2}=a_{1,1,3}=$ $a_{1,2,3}=a_{2,1,1}=a_{2,2,2}=a_{2,3,3}=a_{2,3,1}=a_{2,3,2}=1$; all other components of the matrices $a_{1}$ and $a_{2}$ vanish. Again the beam is not extensible $\left(e_{1,1}=0\right)$ and $b=0$ is a possible solution for the microadjustment. The limit energy reads

$$
\mathscr{E}(u)=\frac{1}{2} \int_{0}^{1}\left(\lambda\left(\frac{\partial^{2} u_{2}}{\partial x_{1}^{2}}\right)^{2}+\zeta\left(\frac{\partial^{2} u_{3}}{\partial x_{1}^{2}}\right)^{2}\right) d x_{1}
$$

(with $\lambda=\frac{1}{3}$ and $\zeta=\frac{1}{2}$ ) and corresponds to a nondegenerate Euler-Bernoulli beam. The bending stiffnesses in the two transverse directions are uncoupled. This is due to the symmetry of our structure.

\subsection{Membranes.}

5.2.1. Regular triangle lattice. The regular triangular truss (see Figure 7) is defined by a cell $Y$ made of only one node $(K=1)$; two vectors $t_{1}=(1,0)$ and $t_{2}=\left(-\frac{1}{2}, \sqrt{3} / 2\right)$ for translating the cell; and five $1 \times 1$ matrices $a_{p}$, defining the interactions between the node of cell $Y_{I}$ and the one of its neighbors $Y_{I+p}$, given by $a_{1}=[0], a_{2}=[1], a_{3}=[1], a_{4}=[1]$, and $a_{5}=[0]$.

We know that the constraint $\boldsymbol{Q} \cdot \nabla u=0$ involves only the symmetric part of $\nabla u$. In terms of $e(u)$ it reads

$$
\frac{\sqrt{3}}{4}\left(\begin{array}{lll}
3 & 1 & 0 \\
1 & 3 & 0 \\
0 & 0 & 4
\end{array}\right) \cdot\left(\begin{array}{l}
e_{1,1}(u) \\
e_{2,2}(u) \\
e_{1,2}(u)
\end{array}\right)=0 .
$$

We already noticed that the matrix $\boldsymbol{Q}$ corresponds to the homogenized behavior which would have been obtained if assuming a smaller order of magnitude for the mechanical interactions. The result above is consistent with this remark and with

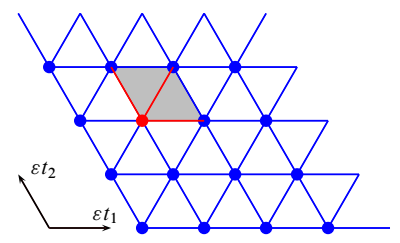

Figure 7. Regular triangle truss. 

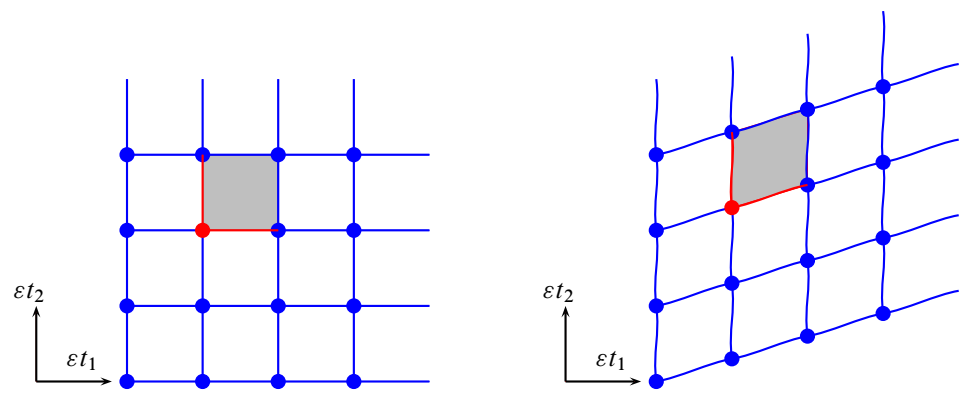

Figure 8. The regular square lattice and its admissible shear deformation.

the result given by [Martinsson and Babuška 2007b]. It corresponds, as expected, to a $2 \mathrm{D}$ isotropic material. Its Lamé coefficients are $\mu=\lambda=\sqrt{3} / 4$, and its Poisson ratio is $v=\frac{1}{3}$. As the matrix above is nondegenerate, the constraint imposes the homogenized material to behave like a rigid body. As we have $\mathscr{E}=0$ for rigid motions, there is no need for supplementary computations for the energy. We get the same uninteresting result for many structures (like, for instance, the Kagome (trihexagonal) lattice studied in [Leung and Guest 2007]). From now on, we will focus only on structures which have more degrees of mobility.

5.2.2. Square grid. The geometry of the regular square lattice (see Figure 8 ) is determined by a single node $(K=1)$, two vectors $t_{1}=(1,0)$ and $t_{2}=(0,1)$ for translating the cell, and five $1 \times 1$ matrices $a_{p}$ defining the interactions between the node $y_{I, 1}^{\varepsilon}$ and its neighbors $y_{I+p, 1}^{\varepsilon}$ given by $a_{1}=[0], a_{2}=[1], a_{3}=[1], a_{4}=[0]$, and $a_{5}=[0]$.

Constraint (22) reads $e_{1,1}(u)=e_{2,2}(u)=0$ : the structure is inextensible in directions $e_{1}$ and $e_{2}$ and only shear is allowed. Microadjustment $b=0$ is optimal, and the limit energy is

$$
\mathscr{E}(u)=\frac{1}{2} \int_{\Omega} \lambda\left(e_{1,2}(u)\right)^{2} d x_{1} d x_{2}
$$

(with $\lambda=6$ ). Contrarily to its $1 \mathrm{D}$ analog, this structure is a classical elastic material. It presents neither any second gradient effect nor generalized continuum effect.

5.2.3. Square grid without constraints. The reader may be frustrated by the fact that almost all our examples present a homogenized behavior subject to strong constraints. We show in this example that constraints can be avoided. Let us replace in the previous example the direct interactions by zigzags (see Figure 9): we consider a cell made of three nodes $y_{1}=(0,0), y_{2}=(0.5,0.3)$, and $y_{3}=(0.3,0.5)$. All components of the five interaction $3 \times 3$ matrices $a_{p}$ vanish but $a_{1,1,2}=a_{1,1,3}=$ $a_{2,2,1}=a_{3,3,1}=1$. 


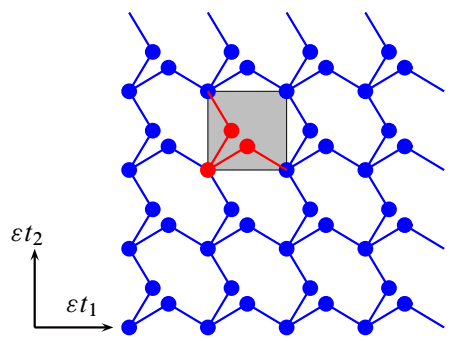

Figure 9. A square structure with unconstrained limit energy.

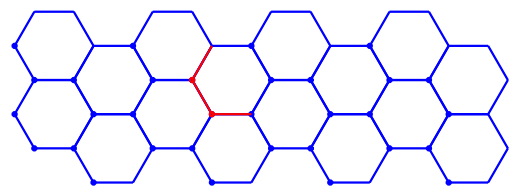

Figure 10. The honeycomb structure.

Constraint (22) disappears, and the limit energy reads

$$
\mathscr{E}(u)=\frac{1}{2} \int_{\Omega}\left(\lambda\left(e_{1,1}(u)\right)^{2}+\lambda\left(e_{2,2}(u)\right)^{2}+\zeta\left(e_{1,2}(u)\right)^{2}\right) d x_{1} d x_{2}
$$

(with $\lambda=\frac{50}{3}$ and $\zeta=3$ ).

In the sequel, for sake of simplicity, we will not try to avoid all constraints: we let the reader check whether a suitable modification of the proposed structures could provide an unconstrained limit energy.

5.2.4. Honeycomb structure. The honeycomb structure (see Figure 10) is frequently put forward for its mechanical properties. It is defined by a cell $Y$ made of two nodes $(K=2)$, two vectors $t_{1}=\left(\frac{3}{2},-\sqrt{3} / 2\right)$ and $t_{2}=(0, \sqrt{3})$ for translating the cell, and five $2 \times 2$ matrices $a_{p}$ defining the interactions between the nodes of cell $Y_{I}$ and the ones of its neighbors $Y_{I+p}$. All their components vanish but $a_{1,1,2}=a_{2,1,2}=a_{3,2,1}=1$. Constraint (22) reads $e_{1,1}(u)+e_{2,2}(u)=0$ : the structure is incompressible. The microadjustment can be eliminated, and the limit energy is

$$
\mathscr{E}(u)=\frac{1}{2} \int_{\Omega} \lambda\|e(u)\|^{2} d x_{1} d x_{2}
$$

(with $\lambda=9$ ). Contrarily to what was expected, this structure is a classical 2D elastic material which presents neither any second gradient effect nor generalized continuum effect. Incompressibility is its only specificity. This geometry has been studied in [Gibson and Ashy 1997; Davini and Ongaro 2011; Davini 2013; Dos Reis and Ganghoffer 2010]. Our result is in concordance with these results but differs due to different assumptions: in these works bending and extensional 

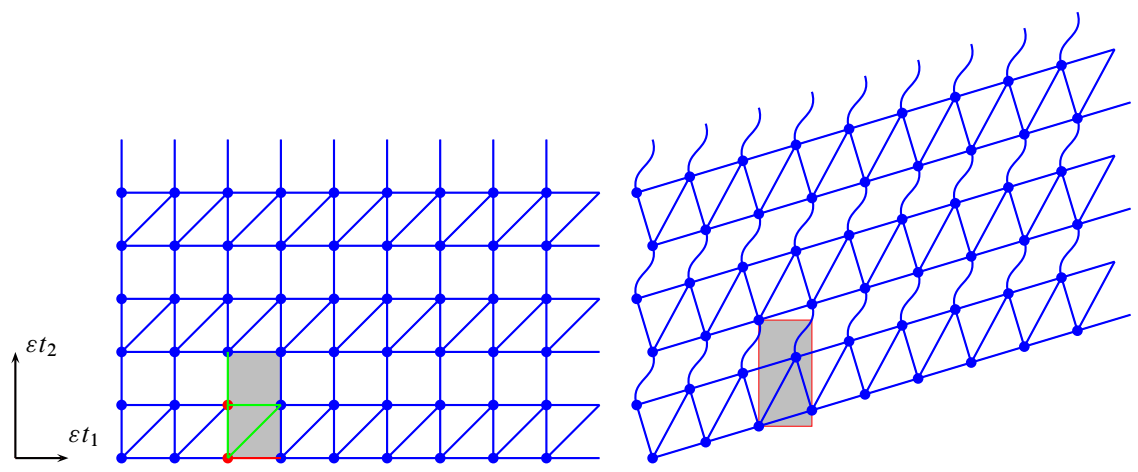

Figure 11. A layered structure.

stiffnesses have the same order of magnitude. It differs also from [Raoult et al. 2008; Le Dret and Raoult 2013] where nonlinearity has been taken into account but where bending stiffness has been chosen either weaker or stronger than we have.

5.2.5. A couple-stress membrane. We add a diagonal bar in one square cell over two in the square lattice described in Section 5.2.2 (see Figure 11). The lattice is now defined by a cell $Y$ made of two nodes $(K=2)$ at points $y_{1}=(0,0)$ and $y_{2}=(0,1)$, the periodicity vectors $t_{1}=(1,0)$ and $t_{2}=(0,2)$, and five $2 \times 2$ matrices $a_{p}$. All components of these matrices vanish but $a_{1,1,2}=a_{2,1,1}=a_{2,2,2}=a_{2,1,2}=$ $a_{3,2,1}=1$.

This structure, when homogenized, is again subject to the constraint $e_{1,1}(u)=$ $e_{2,2}(u)=0$. An optimal microadjustment can be found, and the limit energy reads

$$
\mathscr{E}(u)=\frac{1}{2} \int_{\Omega}\left(\lambda\left(\frac{\partial^{2} u_{2}}{\partial x_{1}^{2}}\right)^{2}+\zeta\left(e_{1,2}(u)\right)^{2}\right) d x_{1} d x_{2}
$$

(with $\lambda=\frac{1}{8}$ and $\zeta=\frac{192}{5}$ ). From the mechanical point of view, the horizontal substructures behave like bending beams and their resistance to bending is responsible for the second gradient part of the limit energy. The model enters the framework of couple-stress models. Indeed, owing to the constraint, the energy can be rewritten

$$
\mathscr{E}(u)=\frac{1}{2} \int_{\Omega}\left(\lambda\left(\frac{\partial}{\partial x_{1}}\left(\frac{\partial u_{2}}{\partial x_{1}}-\frac{\partial u_{1}}{\partial x_{2}}\right)\right)^{2}+\zeta\left(e_{1,2}(u)\right)^{2}\right) d x_{1} d x_{2}
$$

and depends only on the gradient of the skew-symmetric part of the gradient of $u$.

As the energy can alternatively be written $\mathscr{E}(u)=\frac{1}{2} \int_{\Omega}\left(4 \lambda\left(\partial e_{1,2}(u) / \partial x_{1}\right)^{2}+\right.$ $\left.\zeta\left(e_{1,2}(u)\right)^{2}\right) d x_{1} d x_{2}$, the model is clearly endowed with the internal length $\sqrt{4 \lambda / \zeta}$.

5.2.6. Pantographic membrane. This structure is made by a connected array of pantographic structures quite similar to those studied in Section 5.1.3 (see Figures 12 and 13). It is defined by a cell $Y$ made of six nodes $(K=6)$ at points $y_{1}=(0,1)$, 

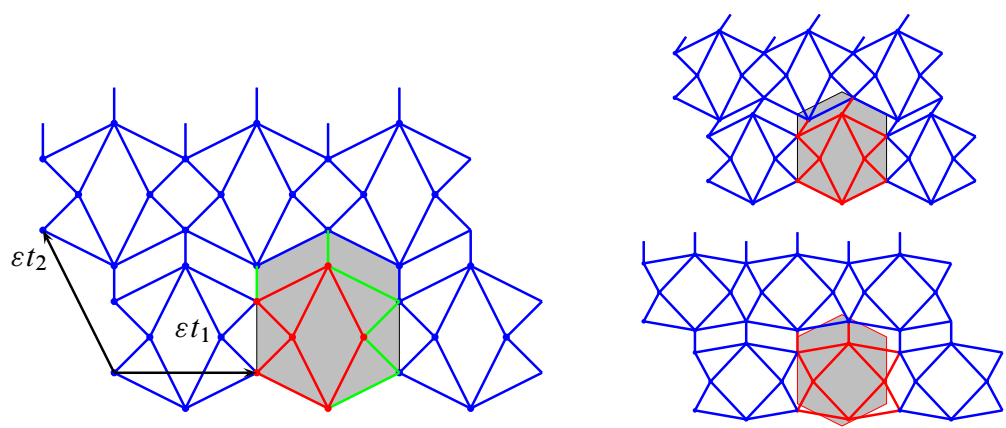

Figure 12. Pantographic membrane and its two admissible deformations (bending of the bars are not represented in the deformed configurations).

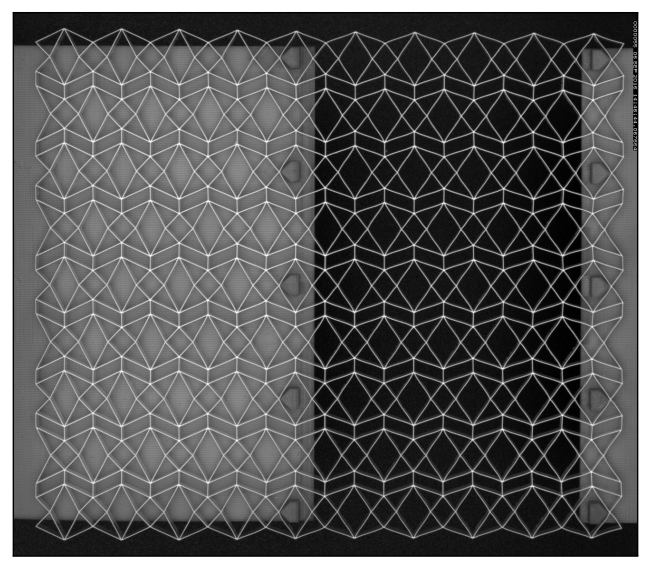

Figure 13. Experiment: traction of a pantographic membrane.

$y_{2}=(0,-1), y_{3}=(1,0), y_{4}=(2,2), y_{5}=(2,-2)$ and $y_{6}=(3,0)$; two vectors $t_{1}=(4,0)$ and $t_{2}=(-2,4)$ for translating the cell; and five $6 \times 6$ matrices $a_{p}$ defining the mechanical interactions. All components of these matrices vanish but

$$
\begin{aligned}
a_{1,1,3}=a_{1,1,4} & =a_{1,2,3}=a_{1,2,5}=a_{1,3,4}=a_{1,3,5}=a_{1,4,6}=a_{1,5,6}=1, \\
a_{2,4,1} & =a_{2,5,2}=a_{2,6,1}=a_{2,6,2}=a_{3,1,5}=a_{4,4,2}=1 .
\end{aligned}
$$

Constraint (22) reads $e_{2,2}(u)=0$. Both horizontal dilatation and shear are admissible. Indeed, these macroscopic displacements, as shown in Figure 12, can be performed without extending any bar. A microadjustment satisfying (26) can be 


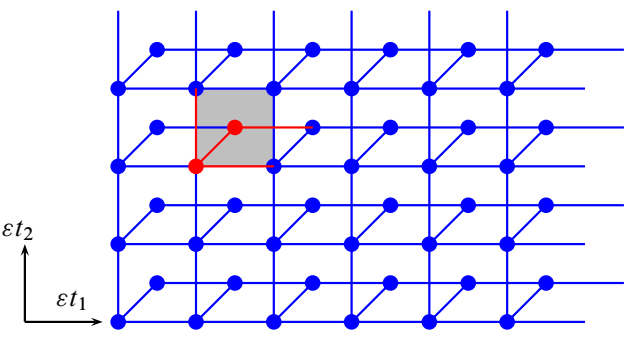

Figure 14. Planar structure leading to Cosserat model.

found, and the homogenized energy is

$$
\begin{aligned}
& \mathscr{E}(u)=\frac{1}{2} \int_{\Omega}\left(\zeta\left(e_{1,1}(u)\right)^{2}+\sigma\left(e_{1,2}(u)\right)^{2}+\right. \mu( \\
&\left(\frac{\partial^{2} u_{1}}{\partial x_{1}^{2}}\right)^{2}+\left(\frac{\partial^{2} u_{2}}{\partial x_{1}^{2}}\right)^{2} \\
&\left.\left.+\lambda\left(\frac{\partial^{2} u_{1}}{\partial x_{1} \partial x_{2}}+\kappa \frac{\partial^{2} u_{2}}{\partial x_{1}^{2}}\right)^{2}\right)\right) d x_{1} d x_{2}
\end{aligned}
$$

(with $\lambda=\frac{484}{131}, \kappa=\frac{13}{44}, \mu=\frac{3}{44}, \sigma=288$, and $\zeta=144$ ). This model which has been studied in [Abdoul-Anziz and Seppecher 2018] is the prototype of complete second gradient models (indeed it does not enter the framework of couple-stress models because of the term $\left.\left(\partial^{2} u_{1} / \partial x_{1}^{2}\right)^{2}\right)$. The very special behavior of this model has been described in [Seppecher et al. 2011]. Due to the strong anisotropy of the structure, it is difficult to distinguish the several intrinsic lengths contained in the model. Structures based on pantographic mechanisms have been intensively studied from theoretical [Placidi et al. 2016] but also numerical [Turco et al. 2017; Harrison 2016] and experimental [Placidi et al. 2017] points of view.

5.2.7. A Cosserat model. We consider the lattice described in Figure 14. It is a planar structure in which we have authorized crossing interactions. The periodic cell is made of two nodes at points $y_{1}=(0,0)$ and $y_{2}=(0.5,0.5)$; the periodicity vectors are $t_{1}=(1,0)$ and $t_{2}=(0,1)$. All components of the five $2 \times 2$ matrices $a_{p}$ vanish but $a_{1,1,2}=a_{2,1,1}=a_{2,2,2}=a_{3,1,1}=1$.

The constraint (22) is again $e_{1,1}(u)=e_{2,2}(u)=0$, and only shear is admissible. The limit energy takes the form

$$
\mathscr{E}(u)=\inf _{\varphi} \frac{1}{2} \int_{\Omega}\left[\zeta\left(\frac{\partial \varphi}{\partial x_{1}}\right)^{2}+\gamma\left(\varphi-\frac{1}{2}\left(\frac{\partial u_{2}}{\partial x_{1}}-\frac{\partial u_{1}}{\partial x_{2}}\right)\right)^{2}+\kappa\left(e_{1,2}(u)\right)^{2}\right] d x_{1} d x_{2}
$$

(with $\zeta=\frac{800}{729}, \gamma=\frac{1600}{333}$, and $\kappa=\frac{56}{9}$ ). The extra variable $\varphi$ plays the role of a Cosserat variable. The reader can understand by considering Figure 14 that the rotation of the bars $\left[y_{I, 1}^{\varepsilon}, y_{I, 2}^{\varepsilon}\right]$ tends to be uniform and that it is coupled to the global displacement owing to the welding of the bars at each node. 


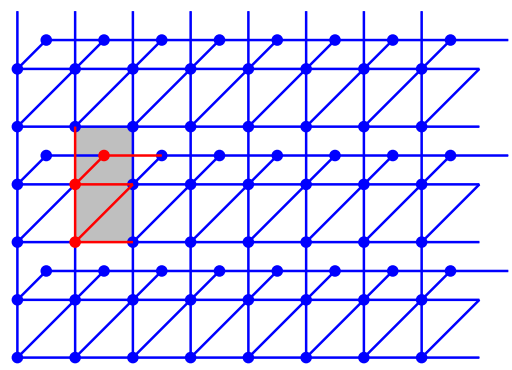

Figure 15. Planar structure leading to both second gradient and Cosserat effects.

5.2.8. Second gradient and Cosserat effects together. Let us combine in Figure 15 the structures of Figures 11 and 14: the periodic cell is now made of three nodes at points $y_{1}=(0,0), y_{2}=(0,1)$, and $y_{3}=(0.5,1.5)$; the periodicity vectors $t_{1}=(1,0)$ and $t_{2}=(0,2)$; and five $3 \times 3$ matrices $a_{p}$. All components of these matrices vanish but $a_{1,1,2}=a_{1,2,3}=a_{2,1,1}=a_{2,1,2}=a_{2,2,2}=a_{2,3,3}=a_{3,2,1}=1$.

The constraint (22) is still $e_{1,1}(u)=e_{2,2}(u)=0$. Shear is admissible. The limit energy takes now the form

$$
\begin{aligned}
\mathscr{E}(u)=\inf _{\varphi} \frac{1}{2} \int_{\Omega}\left[\lambda\left(\frac{\partial^{2} u_{2}}{\partial x_{1}^{2}}\right)^{2}\right. & +\zeta\left(\frac{\partial \varphi}{\partial x_{1}}\right)^{2} \\
& \left.+\gamma\left(\varphi-\frac{1}{2}\left(\frac{\partial u_{2}}{\partial x_{1}}-\frac{\partial u_{1}}{\partial x_{2}}\right)\right)^{2}+\kappa\left(e_{1,2}(u)\right)^{2}\right] d x_{1} d x_{2},
\end{aligned}
$$

thus mixing second gradient and Cosserat effects.

5.3. Plates. Up to now we have only considered planar structures which, of course, are completely degenerate with respect to transverse displacement. Let us now consider structures with a nonzero thickness.

5.3.1. Kirchhoff-Love plate. The considered lattice is made by two superposed regular triangular lattices (see Figure 16). It is defined by a cell $Y$ made of two nodes $(K=2)$ at points $y_{1}=(0,0,0)$ and $y_{2}=(0,0,1)$, the periodicity vectors $t_{1}=(1,0,0)$ and $t_{2}=\left(-\frac{1}{2}, \sqrt{3} / 2,0\right)$, and five $2 \times 2$ matrices $a_{p}$. All components of these matrices vanish but $a_{1,1,2}=a_{2,1,1}=a_{2,2,2}=a_{2,1,2}=a_{2,2,1}=a_{3,1,1}=$ $a_{3,2,2}=a_{3,1,2}=a_{3,2,1}=a_{4,1,1}=a_{4,2,2}=a_{4,1,2}=a_{4,2,1}=1$.

The homogenized model is subject to the constraints $e_{11}(u)=e_{22}(u)=e_{12}(u)=$ 0 (as a membrane, it is undeformable). The microadjustment $b=0$ is optimal, and the limit energy reads

$$
\mathscr{E}(u)=\frac{1}{2} \int_{\Omega}\left(\lambda\left\|\nabla \nabla u_{3}\right\|^{2}+\zeta\left(\Delta u_{3}\right)^{2}\right) d x_{1} d x_{2}
$$




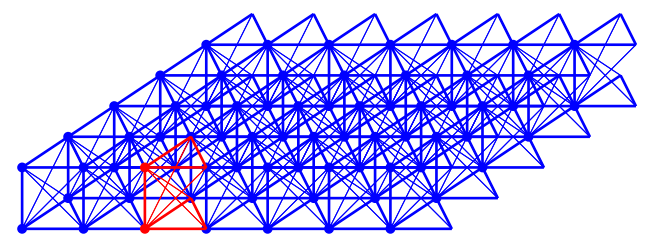

Figure 16. A Kirchoff-Love plate.

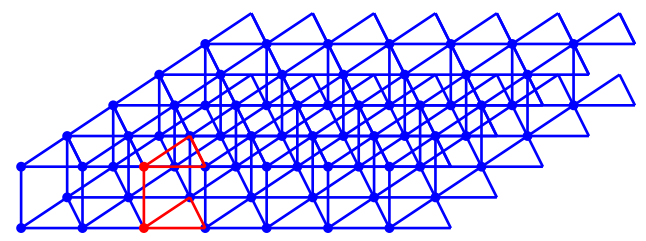

Figure 17. A Mindlin-Reissner plate.

(with $\lambda=\frac{1}{2}$ and $\zeta=\frac{1}{4}$ ) which corresponds to an isotropic inextensible KirchhoffLove bending plate.

5.3.2. Mindlin-Reissner plate. Let us consider the same geometry as in the previous example but where all diagonals joining the lower nodes to the upper ones are deleted (see Figure 17): it is enough to set $a_{2,1,2}=a_{2,2,1}=a_{3,1,2}=a_{3,2,1}=$ $a_{4,1,2}=a_{4,2,1}=0$.

The inextensibility constraint $e_{11}(u)=e_{22}(u)=e_{12}(u)=0$ remains, but now the microadjustment $b$ cannot be completely eliminated. The homogenized energy still involves two extra kinematic variables which can be written $\varphi=\left(\varphi_{1}, \varphi_{2}\right)$ and reads

$$
\mathscr{E}(u)=\inf _{\varphi} \frac{1}{2} \int_{\Omega}\left(\lambda\left\|\nabla u_{3}-\varphi\right\|^{2}+\zeta\|e(\varphi)\|^{2}\right) d x_{1} d x_{2}
$$

(with $\lambda=\frac{9}{4}$ and $\zeta=\frac{1}{2}$ ). This corresponds to an isotropic Mindlin-Reissner plate [Reissner 1985; Sab and Lebée 2015]. Generally, in this theory, $\varphi$ is interpreted as the rotation of the "fiber" which differs from the rotation of the "mid-surface".

5.3.3. Generalized Mindlin-Reissner plate. We are not limited to the extra kinematic variable $\varphi$ introduced in the previous section. We get two such variables when considering three superposed triangular lattices instead of two (see Figure 18), for instance assuming that the lattice is defined by a cell $Y$ made of three nodes at points $y_{1}=(0,0,0), y_{2}=(0,0,1)$, and $y_{3}=(0,0,-2)$ and the periodicity vectors $t_{1}=(1,0,0)$ and $t_{2}=\left(-\frac{1}{2}, \sqrt{3} / 2,0\right)$. All components of the matrices $a_{p}$ vanish but $a_{1,1,2}=a_{2,1,1}=a_{2,2,2}=a_{3,1,1}=a_{3,2,2}=a_{4,1,1}=a_{4,2,2}=a_{1,1,3}=a_{2,3,3}=$ $a_{3,3,3}=a_{4,3,3}=1$. 


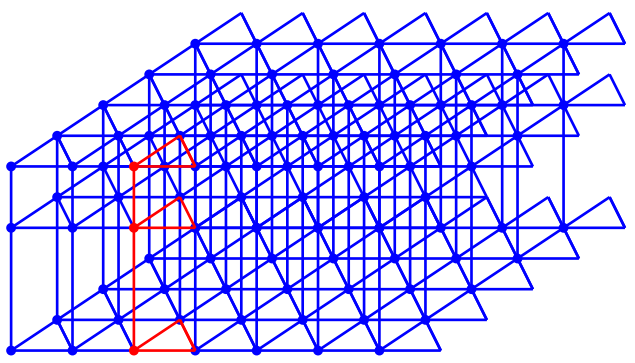

Figure 18. A generalized Mindlin-Reissner plate.

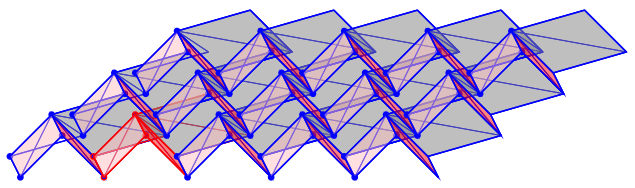

Figure 19. An "origami” plate.

In that case we obtain a model with two "rotation" vectors $\varphi$ and $\psi$ and an energy of type

$$
\mathscr{E}(u)=\inf _{\varphi} \frac{1}{2} \int_{\Omega}\left(\lambda\left\|\nabla u_{3}-\varphi\right\|^{2}+\zeta\left\|\nabla u_{3}-\psi\right\|^{2}+Q(\varphi, \psi, \nabla \varphi, \nabla \psi)\right) d x_{1} d x_{2}
$$

where $Q$ is a nonnegative quadratic form. It is not worth giving here the precise values of $\lambda$ and $\zeta$ nor detailing $Q$.

Multiple layers could also be considered leading to more extra kinematic variables. These models correspond to the generalized Mindlin-Reissner plates recently described in [Lebée and Sab 2017].

Another way for generalizing Reissner models is to mix the structures described in Figures 18 and 14 in order to mix the in-plane Cosserat effect obtained in Section 5.2.7 and Reissner effect. Then we would get a model similar to Reissner but with $\varphi$ living in $\mathbb{R}^{3}$ like described in [Altenbach and Eremeyev 2009].

5.3.4. Origami-type plate. We consider now a lattice made of four nodes at points $y_{1}=(0,0,0), y_{2}=(1,0,1), y_{3}=(-1,1,0)$, and $y_{4}=(0,1,1)$ with periodicity vectors $t_{1}=(2,0,0)$ and $t_{2}=(0,2,0)$; the interaction matrices are defined by $a_{1,1,2}=a_{1,1,3}=a_{1,2,3}=a_{1,2,4}=a_{1,3,4}=a_{2,2,1}=a_{2,2,3}=a_{2,4,1}=a_{2,4,3}=a_{3,3,1}=$ $a_{3,3,2}=a_{3,3,2}=a_{3,4,2}=a_{4,4,1}=a_{5,2,3}=1$ (all other components vanish). This simulates a Miura fold which is suspected to have exotic mechanical properties [Lebée and Sab 2012]: nodes correspond to wedges of the fold while interactions correspond to edges and diagonals of the faces (see Figure 19). 
Constraint (22) reads $e_{1,2}(u)=0$ and $e_{1,1}(u)=e_{2,2}(u)$. Microadjustment $b=0$ is optimal, and the limit energy reads

$$
\mathscr{E}(u)=\frac{1}{2} \int_{\Omega}\left(\lambda\left(\Delta u_{3}\right)^{2}+\zeta\left(e_{1,1}(u)+e_{2,2}(u)\right)^{2}\right) d x_{1} d x_{2}
$$

(with $\lambda=\frac{1}{64}$ and $\zeta=\frac{61}{9}$ ). As a membrane, only isotropic dilatation is admissible and no in-plane second gradient effects are present. As far as transverse displacements are concerned, the structure is degenerated: a curvature is possible with zero elastic energy provided the total curvature vanishes (this behavior is clearly visible when one manipulates this type of fold). From the mathematical point of view, compactness is not ensured and the homogenization result can only be applied when some extra confinement potential is present.

5.3.5. Reinforced origami plate. In the previous example the faces of the structure, made by a planar parallelogram with one diagonal, are very easy to bend. Let us reinforce each of them by adding an out-of-plane node and linking it to the four corners of the face. We add $y_{5}=(0,0,1), y_{6}=(1,0,0), y_{7}=(-1,1,1)$, and $y_{8}=(0,1,0)$, and we add the interactions $a_{1,1,5}=a_{1,2,5}=a_{1,3,5}=a_{1,4,5}=a_{1,2,6}=$ $a_{1,4,6}=a_{2,6,1}=a_{2,6,3}=a_{1,4,8}=a_{1,3,8}=a_{3,8,1}=a_{3,8,2}=a_{1,3,7}=a_{5,2,7}=a_{3,7,1}=$ $a_{2,4,7}=1$. The constraint is unchanged, but the effective energy $\mathscr{E}(u)$ becomes

$$
\begin{aligned}
& \frac{1}{2} \int_{\Omega}\left(\lambda \left(\left(\Delta u_{3}\right)^{2}+\left(\frac{\partial^{2} u_{3}}{\partial x_{1} \partial x_{2}}\right)^{2}\right.\right.\left.+\left(\frac{\partial^{2} u_{2}}{\partial x_{1} \partial x_{2}}\right)^{2}+\left(\frac{\partial^{2} u_{2}}{\partial x_{1}^{2}}\right)^{2}\right) \\
&\left.+\mu\left(\frac{\partial^{2} u_{3}}{\partial x_{1}^{2}}-\frac{\partial^{2} u_{3}}{\partial x_{2}^{2}}\right)^{2}+\zeta\left(e_{1,1}(u)+e_{2,2}(u)\right)^{2}\right) d x_{1} d x_{2}
\end{aligned}
$$

(with $\lambda=\frac{1}{64}, \mu=\frac{1}{192}$, and $\zeta \approx 14.06$ ). Now the plate is nondegenerate: transverse displacement is controlled. As far as in-plane displacement is concerned, the strain tensor takes the form $e=k \operatorname{Id}$ (note that compatibility conditions induce strong constraints for the second derivatives of $k$ ) and the corresponding part of the energy reads

$$
\int_{\Omega}\left(\lambda\left(\frac{\partial k}{\partial x_{1}}\right)^{2}+\zeta k^{2}\right) d x_{1} d x_{2}
$$

The effective membrane is endowed with the intrinsic length $\sqrt{\lambda / \zeta}$.

5.4. Materials. It is difficult to describe clearly and even more to draw periodic lattices with a 3D periodicity. Indeed the number of nodes and edges increases considerably. So we limit ourselves to studying the simple regular cubic lattice and the lattice obtained by replacing each "fiber" of this cubic lattice by a pantographic structure as described in Section 5.1.3. 


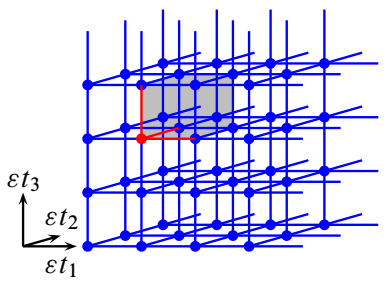

Figure 20. The regular cubic lattice.

5.4.1. Cubic lattice. Let us extend Section 5.2 .2 to dimension 3 by considering a periodic lattice (see Figure 20 ) made by a single node $(K=1)$; three vectors $t_{1}=(1,0,0), t_{2}=(0,1,0)$, and $t_{3}=(0,0,1)$, for translating the cell; and fourteen $1 \times 1$ matrices $a_{p}$ defining the interactions between the node $y_{I, 1}^{\varepsilon}$ and its neighbors $y_{I+p, 1}^{\varepsilon}$ given by $a_{1}=[0], a_{2}=[1], a_{3}=[1], a_{4}=[1]$, and $a_{p}=[0]$ for $p>4$.

Constraint (22) reads $e_{1,1}(u)=e_{2,2}(u)=e_{3,3}(u)=0$ : the structure is inextensible in directions $e_{1}, e_{2}$, and $e_{3}$. Only shear is allowed. Again $b=0$ is an optimal microadjustment, and the limit energy is

$$
\mathscr{E}(u)=\frac{1}{2} \int_{\Omega} \lambda\|e(u)\|^{2} d x_{1} d x_{2} d x_{3}
$$

(with $\lambda=3$ ). This structure is a classical elastic material which presents no second gradient effect nor generalized continuum effect.

5.4.2. Weaved pantographs. We can see the structure of Section 5.4 .1 as made by three families of parallel fibers. Now let us replace the fibers with direction $e_{1}$ by pantographic beams $P_{e_{1}, e_{3}}$ and those with direction $e_{2}$ or $e_{3}$ by pantographic beams $P_{e_{2}, e_{1}}$ or $P_{e_{3}, e_{2}}$, respectively. These beams share the common node $y_{3}=0$, so our new structure is made of a cell containing 16 nodes with 24 internal edges and 12 edges linking it to its neighbors (see Figure 21).

The effective material resulting from the homogenization of this structure is not subject to any constraint. Microadjustment $b=0$ is still optimal, and the limit

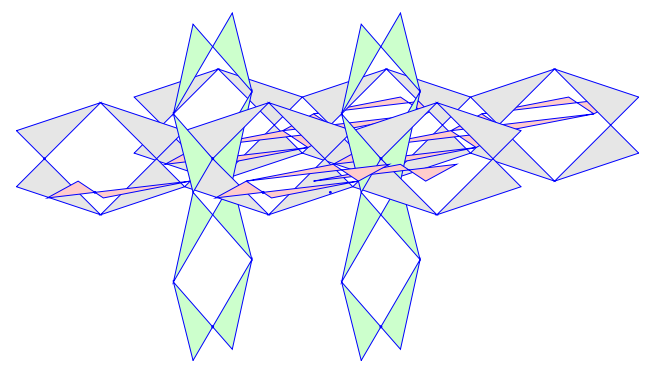

Figure 21. Weaved pantographs. 
energy reads

$$
\begin{aligned}
\mathscr{E}(u)=\frac{1}{2} \int_{\Omega}\left[\lambda \left(\left(\frac{\partial^{2} u_{1}}{\partial x_{1}^{2}}\right)^{2}\right.\right. & \left.+\left(\frac{\partial^{2} u_{2}}{\partial x_{2}^{2}}\right)^{2}+\left(\frac{\partial^{2} u_{3}}{\partial x_{3}^{2}}\right)^{2}\right) \\
& +\mu\left(\left(\frac{\partial^{2} u_{1}}{\partial x_{2}^{2}}\right)^{2}+\left(\frac{\partial^{2} u_{2}}{\partial x_{3}^{2}}\right)^{2}+\left(\frac{\partial^{2} u_{3}}{\partial x_{1}^{2}}\right)^{2}\right) \\
& +\xi\left(\left(e_{3,1}(u)\right)^{2}+\left(e_{2,3}(u)\right)^{2}+\left(e_{1,2}(u)\right)^{2}\right) \\
& \left.+\zeta\left(\left(e_{1,1}(u)\right)^{2}+\left(e_{2,2}(u)\right)^{2}+\left(e_{3,3}(u)\right)^{2}\right)\right] d x_{1} d x_{2} d x_{3},
\end{aligned}
$$

with $\lambda=\frac{2}{23}, \mu=\frac{2}{63}, \zeta=324$, and $\xi \approx 3.91$.

We obtain here a complete strain gradient 3D material. This example illustrates the huge variety of models which can be obtained by homogenizing lattice structures.

\section{Conclusion}

Let us conclude with some remarks.

Our starting point is a lattice made of welded bars with extensional, flexural, and torsional rigidities. The reader could think that, as bending stiffness is by itself a second gradient effect, it is the source of the effective second gradient effects. Surprisingly enough, it is not the case: second gradient effects are due to the extensional stiffness of the bars and to particular designs of the periodic cell while the bending stiffness of the bars is, on the contrary, the source of the first gradient effects in the homogenized energy. Ostoja-Starzewski [2002] has foreseen that lattices can be very useful for giving a micromechanical insight of nonclassical continua, but the role played by the nonextensional part of the mechanical interactions there is overestimated.

Strain gradient and micromorphic models are often presented as competing models. For some researchers, strain gradient models correspond simply to the limit case of micromorphic models in which the coupling between strain and microdeformation is infinitely strong. For other ones, generalized continuum models are regularizations of strain gradient models. Our results show that both effects appear generally together and at the same level.

It is also remarkable that, in our results, strain and strain gradient are never coupled. There is no fundamental reason which prevents such a coupling in a strain gradient model. Some symmetries could explain this absence of coupling [Auffray et al. 2009; Poncelet et al. 2017], but our general homogenization result does not ask for any symmetry in the design of the structure. The point is that strain gradient terms and classical strain terms come from two different sources 
(extensional and flexural/torsional energies) which are assumed at the very beginning to be uncoupled. We think that considering nonhomogeneous or nonisotropic bars would likely lead to coupled models.

The general closure result [Camar-Eddine and Seppecher 2003] allows for effective models more exotic than the ones we have presented in this paper, for instance with an elastic energy depending on the third gradient of the displacement. Indeed we already mentioned that we can design our structures in order to get a degenerate effective energy. In that case, it is natural to rescale again the original energy by multiplying it by $\varepsilon^{-2}$ (or equivalently to act with much lighter forces on the sample), and hope that the limit energy will become nondegenerate. Moreover, one would have to assume that the bars are still slenderer in order to get a compatible flexural energy. In that case, increasing the formal expansion (10) up to order 3, one should likely get third-order models. And, of course, the process can be pushed further. It is not clear whether one can get any reachable effective model by homogenizing frame lattices. Such inverse problem has been addressed in the dynamic case in [Carcaterra et al. 2015].

We have tried to get experimental evidence of second gradient effects (see Figure 13) for the structures described in Section 5.2.6. Up to now, our efforts have been unsuccessful. We think that the major reason for that is twofold: (i) geometrical nonlinearities arise very quickly in these microstructures and (ii) the limit model is extremely sensitive to design; indeed we have checked that a small modification of the position of one node of the periodic cell is enough to change the effective model from strain gradient model to a totally rigid body. Hence, the basic assumption of linear elasticity that current and initial configurations coincide is too strong and the extension of our study to nonlinear elasticity should be undertaken.

\section{Acknowledgments}

The authors thank the French Région Provence-Alpes-Côte-d'Azur and CNRS (Projets Exploratoires Premier Soutien) for their financial support.

\section{References}

[Abdoul-Anziz and Seppecher 2017] H. Abdoul-Anziz and P. Seppecher, "Octave/MATLAB package for homogenizing periodic graph-based elastic structures", software, 2017, available at http:// seppecher.univ-tln.fr/homogenizer/Octave/.

[Abdoul-Anziz and Seppecher 2018] H. Abdoul-Anziz and P. Seppecher, "Homogenization of periodic graph-based elastic structures", J. Éc. Polytech. Math. 5 (2018), 259-288.

[Aifantis 1992] E. C. Aifantis, "On the role of gradients in the localization of deformation and fracture", Int. J. Eng. Sci. 30:10 (1992), 1279-1299.

[Aifantis 1999] E. C. Aifantis, "Strain gradient interpretation of size effects", Int. J. Fracture 95 (1999), 299-314. 
[Alibert and Della Corte 2015] J.-J. Alibert and A. Della Corte, "Second-gradient continua as homogenized limit of pantographic microstructured plates: a rigorous proof", Z. Angew. Math. Phys. 66:5 (2015), 2855-2870.

[Alibert et al. 2003] J.-J. Alibert, P. Seppecher, and F. dell'Isola, "Truss modular beams with deformation energy depending on higher displacement gradients", Math. Mech. Solids 8:1 (2003), $51-73$.

[Allaire 1992] G. Allaire, "Homogenization and two-scale convergence", SIAM J. Math. Anal. 23:6 (1992), 1482-1518.

[Allaire et al. 2016] G. Allaire, M. Briane, and M. Vanninathan, "A comparison between two-scale asymptotic expansions and Bloch wave expansions for the homogenization of periodic structures", SeMA J. 73:3 (2016), 237-259.

[Altenbach and Eremeyev 2009] H. Altenbach and V. A. Eremeyev, "On the linear theory of micropolar plates", Z. Angew. Math. Mech. 89:4 (2009), 242-256.

[Auffray et al. 2009] N. Auffray, R. Bouchet, and Y. Bréchet, "Derivation of anisotropic matrix for bi-dimensional strain-gradient elasticity behavior", Int. J. Solids Struct. 46:2 (2009), 440-454.

[Babuška and Sauter 2004] I. Babuška and S. A. Sauter, "Algebraic algorithms for the analysis of mechanical trusses", Math. Comp. 73:248 (2004), 1601-1622.

[Barbagallo et al. 2017a] G. Barbagallo, A. Madeo, I. Azehaf, I. Giorgio, F. Morestin, and P. Boisse, "Bias extension test on an unbalanced woven composite reinforcement: experiments and modeling via a second-gradient continuum approach”, J. Compos. Mater. 51:2 (2017), 153-170.

[Barbagallo et al. 2017b] G. Barbagallo, A. Madeo, F. Morestin, and P. Boisse, "Modelling the deep drawing of a 3D woven fabric with a second gradient model", Math. Mech. Solids 22:11 (2017), 2165-2179.

[Bellieud 2017] M. Bellieud, "Homogenization of stratified elastic composites with high contrast", SIAM J. Math. Anal. 49:4 (2017), 2615-2665.

[Bellieud and Gruais 2005] M. Bellieud and I. Gruais, "Homogenization of an elastic material reinforced by very stiff or heavy fibers: non-local effects, memory effects", J. Math. Pures Appl. (9) 84:1 (2005), 55-96.

[Bellieud et al. 2016] M. Bellieud, G. Geymonat, and F. Krasucki, "Asymptotic analysis of a linear isotropic elastic composite reinforced by a thin layer of periodically distributed isotropic parallel stiff fibres", J. Elasticity 122:1 (2016), 43-74.

[Bensoussan et al. 1978] A. Bensoussan, J.-L. Lions, and G. Papanicolaou, Asymptotic analysis for periodic structures, Studies in Mathematics and its Applications 5, North-Holland, 1978.

[Bouchitté and Bellieud 2002] G. Bouchitté and M. Bellieud, "Homogenization of a soft elastic material reinforced by fibers", Asymptot. Anal. 32:2 (2002), 153-183.

[Boutin 1996] C. Boutin, "Microstructural effects in elastic composites", Int. J. Solids Struct. 33:7 (1996), 1023-1051.

[Boutin et al. 2017] C. Boutin, F. dell'Isola, I. Giorgio, and L. Placidi, "Linear pantographic sheets: asymptotic micro-macro models identification”, Math. Mech. Complex Syst. 5:2 (2017), 127-162.

[Bouyge et al. 2002] F. Bouyge, I. Jasiuk, S. Boccara, and M. Ostoja-Starzewski, "A micromechanically based couple-stress model of an elastic orthotropic two-phase composite", Eur. J. Mech. A Solid. 21:3 (2002), 465-481.

[Braides 2002] A. Braides, $\Gamma$-convergence for beginners, Oxford Lecture Series in Mathematics and its Applications 22, Oxford University, 2002. 
[Braides and Gelli 2002] A. Braides and M. S. Gelli, "Limits of discrete systems with long-range interactions", J. Convex Anal. 9:2 (2002), 363-399.

[Briane and Camar-Eddine 2007] M. Briane and M. Camar-Eddine, "Homogenization of two dimensional elasticity problems with very stiff coefficients", J. Math. Pures Appl. (9) 88:6 (2007), 483-505.

[Camar-Eddine and Seppecher 2002] M. Camar-Eddine and P. Seppecher, "Closure of the set of diffusion functionals with respect to the Mosco-convergence", Math. Models Methods Appl. Sci. 12:8 (2002), 1153-1176.

[Camar-Eddine and Seppecher 2003] M. Camar-Eddine and P. Seppecher, "Determination of the closure of the set of elasticity functionals", Arch. Ration. Mech. Anal. 170:3 (2003), 211-245.

[Carcaterra et al. 2015] A. Carcaterra, F. dell'Isola, R. Esposito, and M. Pulvirenti, "Macroscopic description of microscopically strongly inhomogenous systems: a mathematical basis for the synthesis of higher gradients metamaterials", Arch. Ration. Mech. Anal. 218:3 (2015), 1239-1262.

[Cherednichenko et al. 2006] K. D. Cherednichenko, V. P. Smyshlyaev, and V. V. Zhikov, "Non-local homogenized limits for composite media with highly anisotropic periodic fibres", Proc. Roy. Soc. Edinburgh Sect. A 136:1 (2006), 87-114.

[Dal Maso 1993] G. Dal Maso, An introduction to $\Gamma$-convergence, Progress in Nonlinear Differential Equations and their Applications 8, Birkhäuser, 1993.

[Davini 2013] C. Davini, "Homogenization of linearly elastic honeycombs", Math. Mech. Solids 18:1 (2013), 3-23.

[Davini and Ongaro 2011] C. Davini and F. Ongaro, "A homogenized model for honeycomb cellular materials", J. Elasticity 104:1-2 (2011), 205-226.

[dell'Isola and Seppecher 1997] F. dell'Isola and P. Seppecher, "Edge contact forces and quasibalanced power", Meccanica 32:1 (1997), 33-52.

[dell'Isola et al. 2015a] F. dell'Isola, U. Andreaus, and L. Placidi, "At the origins and in the vanguard of peridynamics, non-local and higher-gradient continuum mechanics: an underestimated and still topical contribution of Gabrio Piola", Math. Mech. Solids 20:8 (2015), 887-928.

[dell'Isola et al. 2015b] F. dell'Isola, P. Seppecher, and A. Della Corte, "The postulations á la d'Alembert and á la Cauchy for higher gradient continuum theories are equivalent: a review of existing results", P. Roy. Soc. A Math. Phys. 471:2183 (2015), 20150415.

[dell'Isola et al. 2016a] F. dell'Isola, I. Giorgio, M. Pawlikowski, and N. L. Rizzi, "Large deformations of planar extensible beams and pantographic lattices: heuristic homogenization, experimental and numerical examples of equilibrium", P. Roy. Soc. A Math. Phys. 472:2185 (2016), 20150790.

[dell'Isola et al. 2016b] F. dell'Isola, A. Madeo, and P. Seppecher, "Cauchy tetrahedron argument applied to higher contact interactions", Arch. Ration. Mech. Anal. 219:3 (2016), 1305-1341.

[dell'Isola et al. 2017] F. dell'Isola, A. Della Corte, and I. Giorgio, "Higher-gradient continua: the legacy of Piola, Mindlin, Sedov and Toupin and some future research perspectives", Math. Mech. Solids 22:4 (2017), 852-872.

[Dos Reis and Ganghoffer 2010] F. Dos Reis and J. F. Ganghoffer, "Discrete homogenization of architectured materials: implementation of the method in a simulation tool for the systematic prediction of their effective elastic properties", Tech. Mech. 30:1-3 (2010), 85-109.

[Eringen 2001] A. C. Eringen, Microcontinuum field theories, II: Fluent media, Springer, 2001.

[Forest 1999] S. Forest, "Homogenization methods and the mechanics of generalized continua", pp. 35-48 in Geometry, Continua and Microstructure, edited by G. Maugin, Travaux en Cours 60, Hermann, 1999. 
[Germain 1973] P. Germain, Cours de mécanique des milieux continus, tome I: Théorie générale, Masson et Cie, 1973.

[Gibson and Ashy 1997] L. J. Gibson and M. F. Ashy, Cellular solids: structure and properties, 2nd ed., Cambridge University, 1997.

[Giorgio 2016] I. Giorgio, "Numerical identification procedure between a micro-Cauchy model and a macro-second gradient model for planar pantographic structures", Z. Angew. Math. Phys. 67:4 (2016), 95.

[Gonella and Ruzzene 2008] S. Gonella and M. Ruzzene, "Homogenization and equivalent in-plane properties of two-dimensional periodic lattices", Int. J. Solids Struct. 45:10 (2008), 2897-2915.

[Harrison 2016] P. Harrison, "Modelling the forming mechanics of engineering fabrics using a mutually constrained pantographic beam and membrane mesh", Compos. A Appl. S. 81 (2016), 145-157.

[Khruslov 1991] E. Ya. Khruslov, "Homogenized models of composite media”, pp. 159-182 in Composite media and homogenization theory (Trieste, Italy, 1990), edited by G. Dal Maso and G. F. Dell'Antonio, Progress in Nonlinear Differential Equations and their Applications 5, Birkhäuser, 1991.

[Le 2015] D. T. Le, Modèle d'endommagement à gradient: approche par homogénéisation, Ph.D. thesis, Université Pierre et Marie Curie - Paris VI, 2015, available at https://tel.archives-ouvertes.fr/ tel-01367480.

[Le Dret and Raoult 2013] H. Le Dret and A. Raoult, "Homogenization of hexagonal lattices", Netw. Heterog. Media 8:2 (2013), 541-572.

[Lebée and Sab 2012] A. Lebée and K. Sab, "Homogenization of thick periodic plates: application of the bending-gradient plate theory to a folded core sandwich panel", Int. J. Solids Struct. 49:19-20 (2012), 2778-2792.

[Lebée and Sab 2017] A. Lebée and K. Sab, "On the generalization of Reissner plate theory to laminated plates, I: Theory”, J. Elasticity 126:1 (2017), 39-66.

[Leung and Guest 2007] A. C. H. Leung and S. D. Guest, "Single member actuation of kagome lattice structures", J. Mech. Mater. Struct. 2:2 (2007), 303-317.

[Madeo et al. 2017] A. Madeo, A. Della Corte, I. Giorgio, and D. Scerrato, "Modeling and designing micro- and nano-structured metamaterials: towards the application of exotic behaviors", Math. Mech. Solids 22:4 (2017), 873-884.

[Martinsson and Babuška 2007a] P. G. Martinsson and I. Babuška, "Homogenization of materials with periodic truss or frame micro-structures", Math. Models Methods Appl. Sci. 17:5 (2007), 805832.

[Martinsson and Babuška 2007b] P.-G. Martinsson and I. Babuška, "Mechanics of materials with periodic truss or frame micro-structures", Arch. Ration. Mech. Anal. 185:2 (2007), 201-234.

[Mazière and Forest 2015] M. Mazière and S. Forest, "Strain gradient plasticity modeling and finite element simulation of Lüders band formation and propagation", Contin. Mech. Thermodyn. 27:1-2 (2015), 83-104.

[Meunier et al. 2012] N. Meunier, O. Pantz, and A. Raoult, "Elastic limit of square lattices with three-point interactions", Math. Models Methods Appl. Sci. 22:11 (2012), 1250032.

[Milton 2002] G. W. Milton, The theory of composites, Cambridge Monographs on Applied and Computational Mathematics 6, Cambridge University, 2002.

[Mindlin 1963] R. D. Mindlin, "Influence of couple-stresses on stress concentrations", Exp. Mech. 3:1 (1963), 1-7. 
[Mindlin 1965] R. D. Mindlin, "Second gradient of strain and surface-tension in linear elasticity", Int. J. Solids Struct. 1:4 (1965), 417-438.

[Mindlin and Tiersten 1962] R. D. Mindlin and H. F. Tiersten, "Effects of couple-stresses in linear elasticity", Arch. Rational Mech. Anal. 11 (1962), 415-448.

[Nguetseng 1989] G. Nguetseng, "A general convergence result for a functional related to the theory of homogenization”, SIAM J. Math. Anal. 20:3 (1989), 608-623.

[Oleŭnik et al. 1992] O. A. Oleĭnik, A. S. Shamaev, and G. A. Yosifian, Mathematical problems in elasticity and homogenization, Studies in Mathematics and its Applications 26, North-Holland, 1992.

[Ostoja-Starzewski 2002] M. Ostoja-Starzewski, "Lattice models in micromechanics", Appl. Mech. Rev. 55:1 (2002), 35-60.

[Pastukhova 2005] S. E. Pastukhova, "Homogenization of problems of elasticity theory on periodic box and rod frames of critical thickness", J. Math. Sci. 130:5 (2005), 4954-5004.

[Pideri and Seppecher 1997] C. Pideri and P. Seppecher, "A second gradient material resulting from the homogenization of an heterogeneous linear elastic medium", Contin. Mech. Thermodyn. 9:5 (1997), 241-257.

[Placidi et al. 2016] L. Placidi, E. Barchiesi, E. Turco, and N. L. Rizzi, "A review on 2D models for the description of pantographic fabrics", Z. Angew. Math. Phys. 67:5 (2016), 121.

[Placidi et al. 2017] L. Placidi, U. Andreaus, and I. Giorgio, "Identification of two-dimensional pantographic structure via a linear D4 orthotropic second gradient elastic model", J. Engrg. Math. 103 (2017), 1-21.

[Polizzotto and Borino 1998] C. Polizzotto and G. Borino, "A thermodynamics-based formulation of gradient-dependent plasticity", Eur. J. Mech. A Solids 17:5 (1998), 741-761.

[Poncelet et al. 2017] M. Poncelet, N. Auffray, C. Jailin, A. Somera, and C. Morel, "Experimental strain gradient evidence in non-central symmetric lattice", in Generalized and microstructured continua (Arpino, Italy, 2017), EUROMECH Colloquium 579, European Mechanics Society, 2017.

[Rahali et al. 2015] Y. Rahali, I. Giorgio, J. F. Ganghoffer, and F. dell'Isola, "Homogenization à la Piola produces second gradient continuum models for linear pantographic lattices", Internat. J. Engrg. Sci. 97 (2015), 148-172.

[Raoult et al. 2008] A. Raoult, D. Caillerie, and A. Mourad, "Elastic lattices: equilibrium, invariant laws and homogenization”, Ann. Univ. Ferrara Sez. VII Sci. Mat. 54:2 (2008), 297-318.

[Reissner 1985] E. Reissner, "Reflections on the theory of elastic plates", Appl. Mech. Rev. 38:11 (1985), 1453-1464.

[Sab and Lebée 2015] K. Sab and A. Lebée, Homogenization of heterogeneous thin and thick plates, Wiley, 2015.

[Sánchez-Palencia 1980] E. Sánchez-Palencia, Nonhomogeneous media and vibration theory, Lecture Notes in Physics 127, Springer, 1980.

[Sciarra et al. 2007] G. Sciarra, F. dell'Isola, and O. Coussy, "Second gradient poromechanics", Int. J. Solids Struct. 44:20 (2007), 6607-6629.

[Seppecher et al. 2011] P. Seppecher, J.-J. Alibert, and F. dell'Isola, "Linear elastic trusses leading to continua with exotic mechanical interactions", J. Phys. Conf. Ser. 319:1 (2011), 012018.

[Tartar 1989] L. Tartar, "Nonlocal effects induced by homogenization", pp. 925-938 in Partial differential equations and the calculus of variations, vol. II, edited by F. Colombini et al., Progress in Nonlinear Differential Equations and their Applications 2, Birkhäuser, 1989. 
[Tartar 2009] L. Tartar, The general theory of homogenization: a personalized introduction, Lecture Notes of the Unione Matematica Italiana 7, Springer, 2009.

[Toupin 1962] R. A. Toupin, "Elastic materials with couple-stresses", Arch. Rational Mech. Anal. 11 (1962), 385-414.

[Toupin 1964] R. A. Toupin, "Theories of elasticity with couple-stress", Arch. Rational Mech. Anal. 17 (1964), 85-112.

[Triantafyllidis and Aifantis 1986] N. Triantafyllidis and E. C. Aifantis, "A gradient approach to localization of deformation, I: Hyperelastic materials”, J. Elasticity 16:3 (1986), 225-237.

[Turco et al. 2016a] E. Turco, F. dell'Isola, A. Cazzani, and N. L. Rizzi, "Hencky-type discrete model for pantographic structures: numerical comparison with second gradient continuum models", Z. Angew. Math. Phys. 67:4 (2016), 85.

[Turco et al. 2016b] E. Turco, M. Golaszewski, A. Cazzani, and N. L. Rizzi, "Large deformations induced in planar pantographic sheets by loads applied on fibers: experimental validation of a discrete Lagrangian model", Mech. Res. Commun. 76 (2016), 51-56.

[Turco et al. 2017] E. Turco, M. Golaszewski, I. Giorgio, and F. D’Annibale, "Pantographic lattices with non-orthogonal fibres: experiments and their numerical simulations", Compos. B Eng. 118 (2017), 1-14.

[Yang and Misra 2012] Y. Yang and A. Misra, "Micromechanics based second gradient continuum theory for shear band modeling in cohesive granular materials following damage elasticity", Int. J. Solids Struct. 49:18 (2012), 2500-2514.

[Zhikov 2002] V. V. Zhikov, "Homogenization of elasticity problems on singular structures", Izv. Math. 66:2 (2002), 299-365.

[Zhikov and Pastukhova 2003] V. V. Zhikov and S. E. Pastukhova, "Homogenization for elasticity problems on periodic networks of critical thickness", Sb. Math. 194:5 (2003), 697-732.

Received 22 Dec 2017. Revised 27 Mar 2018. Accepted 28 Apr 2018.

Houss AM ABDOUL-ANZIZ: houssam@imath.fr

Institut de Mathématiques de Toulon, Université de Toulon, Toulon, France

PIERRE SEPPECHER: seppecher@imath.fr

Institut de Mathématiques de Toulon, Université de Toulon, Toulon, France 
EDITORIAL BOARD

ANTONIO CARCATERRA

ERIC A. CARLEN

FRANCESCO DELL'ISOLA

RAFFAELE ESPOSITO

ALBERT FANNJIANG

Gilles A. FranCFORT

Pierangelo MARCATI

JEAN-JACQUES MARIGO

PETER A. MARKOWICH

MARTIN OSTOJA-STARZEWSKI

PIERRE SEPPECHER

DAVID J. STEIGMANN

PAUl STEINMANN

PierRe M. SuQueT

MANAGING EDITORS

MICOL AMAR

CORRADO LATTANZIO

ANGELA MADEO

MARTIN OSTOJA-STARZEWSKI

ADVISORY BOARD

ADNAN AKAY

Holm AltenBaCH

MICOL AMAR

HARM ASKES

TEODOR ATANACKOVIĆ

VICTOR BERDICHEVSKY

GUY BOUCHITTÉ

ANDREA BRAIDES

ROBERTO CAMASSA

MAURO CARFORE

ERIC DARVE

FELIX DARVE

ANNA DE MASI

GianPiEtro DEL Piero

EMMANUELE Di BENEDETTO

BERNOLD FIEDLER

IRENE M. GAMBA

DAVID Y. GAO

SERGEY GAVRILYUK

TIMOTHY J. HEALEY

DOMINIQUE JEULIN

ROGER E. KHAYAT

CORRADO LATTANZIO

ROBERT P. LIPTON

ANGELO LUONGO

ANGELA MADEO

JUAN J. MANFREDI

CARLO MARCHIORO

GÉRARD A. MAUGIN

ROBERTO NATALINI PATRIZIO NEFF

ANDREY PIATNITSKI

ERRICO PRESUTTI

MARIO PULVIRENTI

LUCIO RUSSO

Miguel A. F. SANJUAN

PATRICK SElVADURAI

ALEXANDER P. SEYRANIAN

MIROSLAV ŠILHAVÝ

GUIDO SWEERS

ANTOINETTE TORDESILLAS

LEV TRUSKINOVSKY

JUAN J. L. VELÁZQUEZ VINCENZO VESPRI ANGELO VULPIANI msp.org/memocs

Università di Roma "La Sapienza", Italia

Rutgers University, USA

(CO-CHAIR) Università di Roma "La Sapienza", Italia

(TREASURER) Università dell'Aquila, Italia

University of California at Davis, USA

(CO-CHAIR) Université Paris-Nord, France

Università dell'Aquila, Italy

École Polytechnique, France

DAMTP Cambridge, UK, and University of Vienna, Austria

(CHAIR MANAGING EDITOR) Univ. of Illinois at Urbana-Champaign, USA

Université du Sud Toulon-Var, France

University of California at Berkeley, USA

Universität Erlangen-Nürnberg, Germany

LMA CNRS Marseille, France

Università di Roma "La Sapienza", Italia

Università dell'Aquila, Italy

Université de Lyon-INSA (Institut National des Sciences Appliquées), France

(CHAIR MANAGING EDITOR) Univ. of Illinois at Urbana-Champaign, USA

Carnegie Mellon University, USA, and Bilkent University, Turkey

Otto-von-Guericke-Universität Magdeburg, Germany

Università di Roma "La Sapienza", Italia

University of Sheffield, UK

University of Novi Sad, Serbia

Wayne State University, USA

Université du Sud Toulon-Var, France

Università di Roma Tor Vergata, Italia

University of North Carolina at Chapel Hill, USA

Università di Pavia, Italia

Stanford University, USA

Institut Polytechnique de Grenoble, France

Università dell'Aquila, Italia

Università di Ferrara and International Research Center MEMOCS, Italia

Vanderbilt University, USA

Freie Universität Berlin, Germany

University of Texas at Austin, USA

Federation University and Australian National University, Australia

Université Aix-Marseille, France

Cornell University, USA

École des Mines, France

University of Western Ontario, Canada

Università dell' Aquila, Italy

Louisiana State University, USA

Università dell'Aquila, Italia

Université de Lyon-INSA (Institut National des Sciences Appliquées), France University of Pittsburgh, USA

Università di Roma "La Sapienza”, Italia

Université Paris VI, France

Istituto per le Applicazioni del Calcolo "M. Picone", Italy

Universität Duisburg-Essen, Germany

Narvik University College, Norway, Russia

Università di Roma Tor Vergata, Italy

Università di Roma "La Sapienza”, Italia

Università di Roma “Tor Vergata”, Italia

Universidad Rey Juan Carlos, Madrid, Spain

McGill University, Canada

Moscow State Lomonosov University, Russia

Academy of Sciences of the Czech Republic

Universität zu Köln, Germany

University of Melbourne, Australia

École Polytechnique, France

Bonn University, Germany

Università di Firenze, Italia

Università di Roma La Sapienza, Italia

MEMOCS (ISSN 2325-3444 electronic, 2326-7186 printed) is a journal of the International Research Center for the Mathematics and Mechanics of Complex Systems at the Università dell'Aquila, Italy.

Cover image: "Tangle” by $\odot$ John Horigan; produced using the Context Free program (contextfreeart.org).

PUBLISHED BY

7 mathematical sciences publishers

nonprofit scientific publishing

http://msp.org/

(C) 2018 Mathematical Sciences Publishers 
Mathematics and Mechanics of Complex Systems vol. 6 no. 3

The variational structure of classical plasticity

Gianpietro Del Piero

Far-reaching Hellenistic geographical knowledge hidden in

Ptolemy's data

\section{Lucio Russo}

Generation of SH-type waves due to shearing stress discontinuity in an anisotropic layer overlying an initially stressed elastic half-space

Santosh Kumar and Dinbandhu Mandal

Strain gradient and generalized continua obtained by homogenizing frame lattices

Houssam Abdoul-Anziz and Pierre Seppecher

On the effect of phase transition on the manifold dimensionality: application to the Ising model

Elena Lopez, Adrien Scheuer, Emmanuelle Abisset-Chavanne and Francisco Chinesta

MEMOCS is a journal of the International Research Center for the Mathematics and Mechanics of Complex Systems at the Università dell' Aquila, Italy.

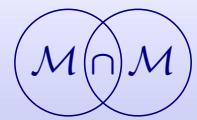

